\title{
Efficient Synthesis of the 3-Benzazepine Framework via Intramolecular Heck Reductive Cyclization
}

\author{
Pavel A. Donets, Erik V. Van der Eycken* \\ Laboratory for Organic \& Microwave-Assisted Chemistry (LOMAC), Department of Chemistry, \\ University of Leuven, Celestijnenlaan 200F, B-3001 Leuven
}

* To whom correspondence should be addressed. E-mail: erik.vandereycken@chem.kuleuven.be

\section{Contents}

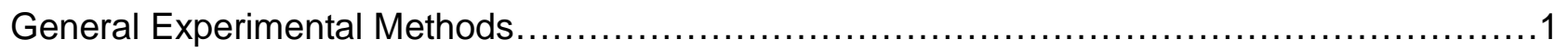

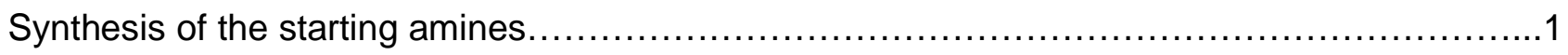

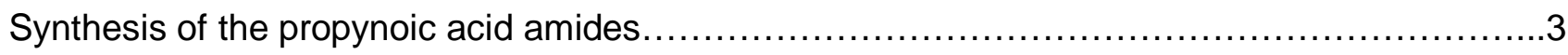

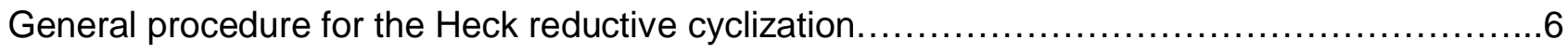

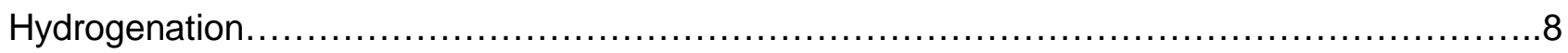

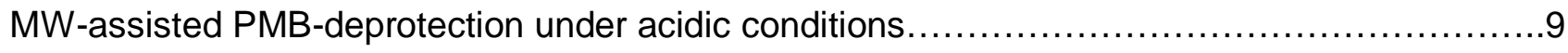

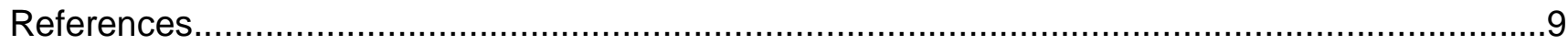

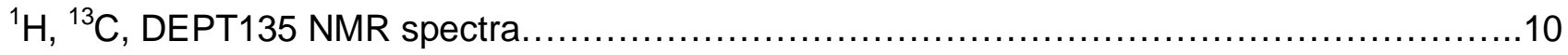




\section{General Experimental Methods.}

${ }^{1} \mathrm{H}$ and ${ }^{13} \mathrm{C}$ NMR spectra were recorded on Bruker Avance 300 or Bruker 400 instruments. The ${ }^{1} \mathrm{H}$ and ${ }^{13} \mathrm{C}$ chemical shifts are reported in parts per million relative to tetramethylsilane using the residual solvent signal as the internal reference. Mass spectra were recorded on Kratos MS50TC and Kratos Mach III system. The ion source temperature was $150-250^{\circ} \mathrm{C}$, as required. High-resolution El-mass spectra were recorded with a resolution of 10000 . The low-resolution spectra were obtained with HP5989A MS instrument. For thin-layer chromatography, analytical TLC plates (Alugram SIL G/UV254 and 70-230 mesh silica gel (E. M. Merck) were used.

\section{Microwave Irradiation Experiments.}

A multimode Milestone MicroSYNTH microwave reactor (Laboratory Microwave Systems) was used in the standard configuration as delivered, including proprietary software. All experiments were carried out in sealed microwave process vials $(15 \mathrm{~mL})$. After completion of the reaction, the vial was cooled down to $25{ }^{\circ} \mathrm{C}$ via air jet cooling before opening.

\section{Synthesis of the starting amines:}

[2-(2-Bromo-4,5-dimethoxyphenyl)ethyl]-(4-methoxybenzyl)amine

(2a),

[2-(2-Bromo-4,5dimethoxyphenyl)ethyl]-(4-methoxybenzyl)methylamine (2b), 2-(2-Bromo-4,5-dimethoxyphenyl)ethylamine (2c) were synthesized according to the references 1,2 and 3 respectively.<smiles>CNCCc1ccccc1Br</smiles>

[2-(2-Bromophenyl)ethyl]methylamine (2d) was synthesized following the known procedure ${ }^{2}$. 2-(2Bromophenyl)ethylamine $2 \mathrm{~g}(10 \mathrm{mmol})$ and benzaldehyde $1.061 \mathrm{~g}(10 \mathrm{mmol})$ in toluene $(50 \mathrm{~mL})$ were refluxed with azeotropic water removal during $4 \mathrm{~h}$. After cooling to $90^{\circ} \mathrm{C} \mathrm{Me}_{2} \mathrm{SO}_{4} 0.95 \mathrm{~mL}(10 \mathrm{mmol})$ was added and heating was continued for $12 \mathrm{~h}$. Upon cooling to ambient temperature aqueous $10 \% \mathrm{HCl}(30 \mathrm{~mL})$ was added and the mixture was stirred for $1 \mathrm{~h}$. Further aqueous layer was washed with DCM, basified with $\mathrm{NaOH}$ pellets to $\mathrm{pH} 10$ and extracted again with DCM. Drying with $\mathrm{Na}_{2} \mathrm{SO}_{4}$ and concentration under reduced pressure gave the pure methylated amine $1.930 \mathrm{~g}$ (84\%) as a colourless oil. (DCM / MeOH / $\left.\mathrm{NH}_{3}(\mathrm{aq}) 150 / 15 / 0.5\right)\left(R_{f} 0.26\right) ;{ }^{1} \mathrm{H} \mathbf{N M R}\left(\mathrm{CDCl}_{3}, 300 \mathrm{MHz}\right): \delta 7.53(\mathrm{~d}, 1 \mathrm{H}$, $J=7.9 \mathrm{~Hz}), 7.23(\mathrm{~m}, 2 \mathrm{H}), 7.02-7.10(\mathrm{~m}, 1 \mathrm{H}), 2.80-3.00(\mathrm{~m}, 4 \mathrm{H}), 2.47(\mathrm{~s}, 3 \mathrm{H}), 1.26(\mathrm{bs}, 1 \mathrm{H}) ;{ }^{13} \mathrm{C} \mathrm{NMR}\left(\mathrm{CDCl}_{3}, 75 \mathrm{MHz}\right)$ : $\delta$ 139.61, 133.30, 131.17, 128.28, 127.85, 124.98, 51.97, 36.86, 36.76; LRMS (Cl) (m/z, relative intensity) : 214 / $216\left([\mathrm{M}+\mathrm{H}]^{+}, 100\right), 183 / 185(44), 134(19)$.<smiles>CC(C)NCCc1ccccc1Br</smiles>

[2-(2-Bromophenyl)ethyl]isopropylamine (2e). 2-(2-Bromophenyl)ethylamine $1 \mathrm{~g}(5 \mathrm{mmol})$ and anhydrous acetone $348 \mathrm{mg}$ (1.2 equiv) in DCE $(5 \mathrm{~mL})$ were stirred at ambient temperature for $2 \mathrm{~h}$. Then freshly prepared $\mathrm{NaBH}(\mathrm{OAc})_{3} 318$ $\mathrm{mg}$ (1.5 equiv) was added and stirring was continued. Another portion of $\mathrm{NaBH}(\mathrm{OAc})_{3} 318 \mathrm{mg}$ (1.5 equiv) was added after $8 \mathrm{~h}$ and the reaction was left with stirring overnight. Afterwards the reaction mixture was diluted with DCM and washed with $1 \mathrm{M} \mathrm{NaOH}$. The organic layer was dried $\left(\mathrm{Na}_{2} \mathrm{SO}_{4}\right)$, concentrated and applied to a silica gel column. Elution with $\mathrm{DCM} / \mathrm{MeOH} / \mathrm{NH}_{3}(\mathrm{aq})(150 / 15 / 1)\left(R_{f} 0.40\right)$ afforded pure isopropylated amine $847 \mathrm{mg}(70 \%)$ as colourless oil. ${ }^{1} \mathbf{H}$ NMR $\left(\mathrm{CDCl}_{3}, 300 \mathrm{MHz}\right): \delta 7.54(\mathrm{~d}, 1 \mathrm{H}, J=7.7 \mathrm{~Hz}), 7.26(\mathrm{~m}, 1 \mathrm{H}), 7.00-7.14(\mathrm{~m}, 1 \mathrm{H}), 2.80-3.00(\mathrm{~m}$, $5 \mathrm{H}), 1.40-2.00$ (bs, $1 \mathrm{H}), 1.09$ (d, $6 \mathrm{H}, J=5.5 \mathrm{~Hz}) ;{ }^{13} \mathrm{C}$ NMR $\left(\mathrm{CDCl}_{3}, 75 \mathrm{MHz}\right): 139.82,133.30,131.11,128.30,127.87$, 124.98, 48.88, 47.43, 37.34, 23.29; LRMS (Cl) (m/z, relative intensity) : 242 / $246\left([\mathrm{M}+\mathrm{H}]^{+}, 100\right), 226 / 228(26), 183 /$ 185 (12), $162(19), 72(60), 59(38)$.

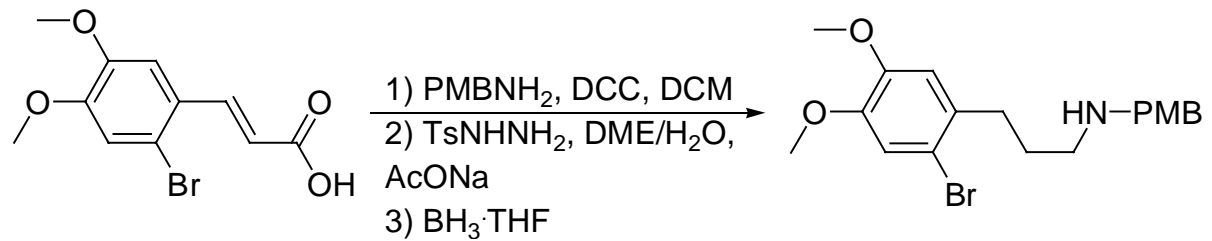


1) 3-(2-Bromo-4,5-dimethoxyphenyl)-N-(4-methoxybenzyl)acrylamide. 2-Bromo-4,5-dimethoxycinnamic acid $2.01 \mathrm{~g}(7 \mathrm{mmol})$ and p-metoxybenzylamine $947 \mathrm{mg}(6.9 \mathrm{mmol})$, DMAP $86 \mathrm{mg}(10 \mathrm{~mol} \%)$ were all mixed up in dry DCM $(50 \mathrm{~mL})$. DCC $1.465 \mathrm{mg}(7.1 \mathrm{mmol})$ was added and the reaction mixture was stirred at ambient temperature overnight. Afterwards the precipitate of dicyclohexylurea was filtered off and washed well with EtOAc. Silica was added to the filtrate and the solvent was removed under reduced pressure. The resulting powder was applied to a silica gel column. Elution with $\mathrm{DCM} / \mathrm{Et}_{2} \mathrm{O}(4 / 1)\left(R_{f} 0.43\right)$ afforded the required amide $2.749 \mathrm{~g}(98 \%)$ slightly contaminated with dicyclohexylurea. ${ }^{1} \mathrm{H}$ NMR (DMSO- $\left.d_{6}, 300 \mathrm{MHz}\right): \delta 8.50(\mathrm{bs}, 1 \mathrm{H}), 7.64(\mathrm{~d}, 1 \mathrm{H}, J=14.9 \mathrm{~Hz}), 7.10-7.25(\mathrm{~m}, 4 \mathrm{H}), 6.89$ $(\mathrm{d}, 2 \mathrm{H}, J=7.9 \mathrm{~Hz}), 6.63(\mathrm{~d}, 1 \mathrm{H}, J=14.9 \mathrm{~Hz}), 4.31(\mathrm{~s}, 2 \mathrm{H}), 3.81(\mathrm{~s}, 6 \mathrm{H}), 3.73(\mathrm{~s}, 3 \mathrm{H}) ;{ }^{13} \mathrm{C} \mathrm{NMR}\left(\mathrm{DMSO}-d_{6}, 75 \mathrm{MHz}\right): \delta$ $164.60,158.27,150.62,148.52,136.60,131.25,128.79,126.22,123.12,115.59,113.72,109.41,56.01,55.58$, $55.06,41.87$.

2) 3-(2-Bromo-4,5-dimethoxyphenyl)-N-(4-methoxybenzyl)propionamide. 3-(2-Bromo-4,5-dimethoxyphenyl)-N-(4methoxybenzyl)acrylamide $2.719 \mathrm{~g}(6.69 \mathrm{mmol})$ and $\mathrm{TsNHNH}_{2} 12.5 \mathrm{~g}$ (10 equiv) were dissolved in DME (120 mL) and the solution was brought to reflux. Solution of AcONa $9.6 \mathrm{~g}$ (17.5 equiv) in water $(70 \mathrm{~mL})$ was added dropwise to the boiling reaction mixture during $2 \mathrm{~h}$. After cooling to ambient temperature the solvent was removed under reduced pressure. The residue was dissolved in DCM and washed successively with $1 \mathrm{M} \mathrm{NaOH}, 1 \mathrm{M} \mathrm{HCl}$ and water. The organic layer was dried $\left(\mathrm{MgSO}_{4}\right)$, concentrated. The product was isolated by column chromatography on silica gel eluting with DCM / $\mathrm{Et}_{2} \mathrm{O}(4 / 1)\left(R_{f} 0.43\right)$. Yield: $2.420 \mathrm{~g}(89 \%) .{ }^{1} \mathrm{H}$ NMR (DMSO- $\left.d_{6}, 300 \mathrm{MHz}\right): \delta 8.28$ (bs, $\left.1 \mathrm{H}\right), 7.04-$ $7.12(\mathrm{~m}, 3 \mathrm{H}), 6.90(\mathrm{~s}, 1 \mathrm{H}), 8.83(\mathrm{~d}, 2 \mathrm{H}, J=8.4 \mathrm{~Hz}), 4.18(\mathrm{~d}, 2 \mathrm{H}, J=5.3 \mathrm{~Hz}), 3.74(\mathrm{~s}, 3 \mathrm{H}), 3.72(\mathrm{~s}, 3 \mathrm{H}), 3.68(\mathrm{~s}, 3 \mathrm{H})$, $2.87(\mathrm{t}, 2 \mathrm{H}, J=7.7 \mathrm{~Hz}), 2.41(\mathrm{t}, 2 \mathrm{H}, J=7.7 \mathrm{~Hz}) ;{ }^{13} \mathrm{C}$ NMR (DMSO-d, $\left.75 \mathrm{MHz}\right): \delta 170.79,158.15,148.19,147.89$, $132.08,131.47,128.48,115.50,113.59,113.13,55.86,55.56,55.03,35.52,31.03$.

3) [3-(2-Bromo-4,5-dimethoxyphenyl)propyl]-(4-methoxybenzyl)amine. 3-(2-Bromo-4,5-dimethoxyphenyl)-N-(4methoxybenzyl)propionamide $2.420 \mathrm{~g}(5.93 \mathrm{mmol})$ was dissolved in dry THF (20 mL) under argon atmosphere and the solution was cooled to $0^{\circ} \mathrm{C} .1 \mathrm{M}$ solution of $\mathrm{BH}_{3} \mathrm{THF}$ complex $11.86 \mathrm{~mL}$ (2 equiv) was added via cannula and after $30 \mathrm{~min}$ of stirring at ambient temperature the reaction mixture was refluxed overnight. Afterwards $3 \mathrm{M} \mathrm{HCl}(8 \mathrm{~mL})$ was added dropwise and the solution was boiled for $30 \mathrm{~min}$. THF was evaporated under reduced pressure, the resulting mixture was basified with $\mathrm{NaOH}$ pellets till $\mathrm{pH} \sim 10$, extracted with $\mathrm{DCM}$. The organic layer was dried $\left(\mathrm{Na}_{2} \mathrm{SO}_{4}\right)$ and concentrated. The required amine was isolated by column chromatography on silica gel eluting with $\mathrm{DCM} / \mathrm{MeOH} /$ $\mathrm{NH}_{3}(\mathrm{aq})(150 / 15 / 0.5)\left(R_{\mathrm{f}} 0.38\right)$. Yield: $2.016 \mathrm{~g}(86 \%) .{ }^{1} \mathrm{H} \mathbf{N M R}\left(\mathrm{CDCl}_{3}, 400 \mathrm{MHz}\right): \delta 7.23(\mathrm{~d}, 2 \mathrm{H}, J=8.7 \mathrm{~Hz}), 6.98$ $(\mathrm{s}, 1 \mathrm{H}), 6.85(\mathrm{~d}, 2 \mathrm{H}, J=8.7 \mathrm{~Hz}), 6.71(\mathrm{~s}, 1 \mathrm{H}), 3.63(\mathrm{~s}, 3 \mathrm{H}), 3.62(\mathrm{~s}, 3 \mathrm{H}), 3.79(\mathrm{~s}, 3 \mathrm{H}), 3.73(\mathrm{~s}, 2 \mathrm{H}), 2.66-2.73(\mathrm{~m}, 4 \mathrm{H})$, $1.80(\mathrm{~m}, 2 \mathrm{H}) ;{ }^{13} \mathrm{C}$ NMR $\left(\mathrm{CDCl}_{3}, 100 \mathrm{MHz}\right): \delta 159.01,148.72,148.16,133.88,133.03,129.70,129.43,128.64$, 115.91, 114.35, 114.17, 113.32, 56.54, 56.41, 55.65, 53.85, 49.13, 33.95, 30.98; LRMS (CI) (m/z, relative intensity) : $394 / 396\left([\mathrm{M}+\mathrm{H}]^{+}, 24\right), 314(34), 286$ / $288(12), 225(9), 149(10), 121(100)$.

Synthesis of the propynoic acid amides (3a-0,10a,b). $\left({ }^{1} \mathrm{H}\right.$ and ${ }^{13} \mathrm{C}$ NMR spectra are described for both rotamers on the amide bond ( $E / Z$ ratio in most cases $1 / 1$, unless specified):

3-Phenylpropynoic acid [2-(2-bromo-4,5-dimethoxyphenyl)ethyl]-(4-methoxybenzyl)amide (3a). Amine 2a $3.8 \mathrm{~g}$ $(10 \mathrm{mmol})$ dry $\mathrm{Et}_{3} \mathrm{~N} 1.67 \mathrm{~mL}$ (1.2 equiv), DMAP $366 \mathrm{mg}(10 \mathrm{~mol} \%)$ were mixed up in dry DCM $(50 \mathrm{~mL})$ under argon atmosphere and the mixture was cooled to $0^{\circ} \mathrm{C}$. Phenylpropionic acid chloride $1.810 \mathrm{~g}$ (1.1 equiv) dissolved in DCM $(15 \mathrm{~mL})$ was added dropwise during $1 \mathrm{~h}$. After completion of the addition the mixture was stirred at ambient temperature for $40 \mathrm{~min}$ more. Then DCM was added and the organic layer was washed successively with $1 \mathrm{M} \mathrm{HCl}$, $\mathrm{NaHCO}_{3}$ saturated solution and water. After drying $\left(\mathrm{MgSO}_{4}\right)$ and concentration the residue was applied to a silica gel column. Elution with DCM / MeOH $(80 / 1)\left(R_{f} 0.32\right)$ afforded the product $4.762 \mathrm{~g}(94 \%)$ as a transparent viscous oil, which further solidified on standing. ${ }^{1} \mathrm{H}$ NMR $\left(\mathrm{CDCl}_{3}, 400 \mathrm{MHz}\right): \delta 7.49-7.55(\mathrm{~m}, 4 \mathrm{H}), 7.31-7.43(\mathrm{~m}, 6 \mathrm{H}), 7.26(\mathrm{~d}, 2 \mathrm{H}$, $J=8.3 \mathrm{~Hz}), 7.23(\mathrm{~d}, 2 \mathrm{H}, J=8.3 \mathrm{~Hz}), 6.98(\mathrm{~s}, 1 \mathrm{H}), 6.91(\mathrm{~s}, 1 \mathrm{H}), 6.87(\mathrm{~d}, 4 \mathrm{H}, J=8.3 \mathrm{~Hz}), 6.76(\mathrm{~s}, 1 \mathrm{H}), 6.54(\mathrm{~s}, 1 \mathrm{H})$, $4.62(\mathrm{~s}, 2 \mathrm{H}), 4.60(\mathrm{~s}, 2 \mathrm{H}), 3.85(\mathrm{~s}, 3 \mathrm{H}), 3.84(\mathrm{~s}, 3 \mathrm{H}), 3.79(\mathrm{~s}, 6 \mathrm{H}), 3.77(\mathrm{~s}, 3 \mathrm{H}), 3.70-3.75(\mathrm{~m}, 2 \mathrm{H}), 3.64(\mathrm{~s}, 3 \mathrm{H}), 3.50-$ $3.56(\mathrm{~m}, 2 \mathrm{H}), 2.92-3.00(\mathrm{~m}, 4 \mathrm{H}) ;{ }^{13} \mathrm{C}$ NMR $\left(\mathrm{CDCl}_{3}, 75 \mathrm{MHz}\right): \delta 159.77,159.62,155.1,154.96,148.94,148.87$, $148.71,132.84,132.69,130.53,130.43,130.35,129.86,129.55,129.22,128.93,128.73,121.00,120.78,116.01$, $115.78,114.57,114.44,113.99,113.78,90.53,90.16,82.37,82.12,56.53,56.27,55.67,53.34,48.53,47.88,44.72$, $35.61,33.60$.

\section{General procedure ${ }^{4}$ for the synthesis of propynoic acid amides:}




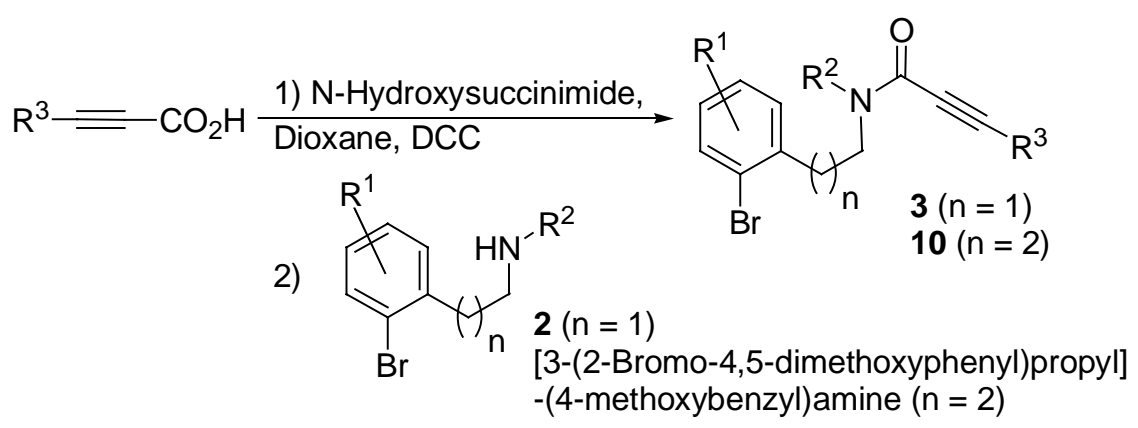

3-substituted 2-propynoic acid (1.1 equiv) and $\mathrm{N}$-hydroxysuccinimide $127 \mathrm{mg}$ (1.1 equiv) were dissolved in dry dioxane (10 mL). DCC $217 \mathrm{mg}$ (1.05 equiv) was added and the reaction was stirred at ambient temperature for 4-5h. Amine (1 mmol, 1 equiv) dissolved in dioxane $(5 \mathrm{~mL})$ was added in one portion and the reaction was left with stirring overnight. The precipitate was filtered off and washed well with $\mathrm{Et}_{2} \mathrm{O}$. The organic layer was washed with brine, dried $\left(\mathrm{MgSO}_{4}\right)$ and concentrated. Pure amides were isolated by column chromatography on silica gel.

2-Butynoic acid [2-(2-bromo-4,5-dimethoxyphenyl)ethyl]-(4-methoxybenzyl)amide (3b). Synthesized from 2butynoic acid $92 \mathrm{mg}$ and amine $2 \mathrm{a} 380 \mathrm{mg}$. Yield: $361 \mathrm{mg}(81 \%)$. ${ }^{1} \mathrm{H}$ NMR $\left(\mathrm{CDCl}_{3}, 300 \mathrm{MHz}\right): \delta 7.12-7.24(\mathrm{~m}, 4 \mathrm{H})$, $6.97(\mathrm{~d}, 2 \mathrm{H}, J=5.7 \mathrm{~Hz}), 6.81-6.90(\mathrm{~m}, 4 \mathrm{H}), 6.73(\mathrm{~s}, 1 \mathrm{H}), 6.54(\mathrm{~s}, 1 \mathrm{H}), 4.52(\mathrm{~s}, 4 \mathrm{H}), 3.77-3.88(\mathrm{~m}, 18 \mathrm{H}), 3.65(\mathrm{t}, 2 \mathrm{H}, J=$ $7.1 \mathrm{~Hz}), 3.44(\mathrm{t}, 2 \mathrm{H}, J=7.1 \mathrm{~Hz}), 2.90(\mathrm{t}, 4 \mathrm{H}, J=7.1 \mathrm{~Hz}), 2.00(\mathrm{~s}, 3 \mathrm{H}), 1.98(\mathrm{~s}, 3 \mathrm{H}) ;{ }^{13} \mathrm{C} \mathrm{NMR}\left(\mathrm{CDCl}_{3}, 75 \mathrm{MHz}\right): \delta$ $159.68,159.52,155.26,155.11,148.89,148.62,130.47,130.24,130.00,129.46,129.28,128.85,115.93,115.71$, $114.78,114.69,114.47,114.38,113.95,89.37,89.08,74.05,73.78,56.59,56.51,55.68,53.12,48.16,47.67,44.37$, $35.45,33.50,4.60,4.50$.

3-Trimethylsilylpropynoic acid [2-(2-bromo-4,5-dimethoxyphenyl)ethyl]-(4-methoxybenzyl)amide (3c). Synthesized from trimethylsilylpropynoic acid $156 \mathrm{mg}$ and amine $2 \mathrm{a} 380 \mathrm{mg}$. Yield: $504 \mathrm{mg}(100 \%)$. ${ }^{1} \mathbf{H ~ N M R}\left(\mathrm{CDCl}_{3}\right.$, $300 \mathrm{MHz}): \delta 7.15-7.25(\mathrm{~m}, 4 \mathrm{H}), 6.98(\mathrm{~d}, 2 \mathrm{H}, \mathrm{J}=7.7 \mathrm{~Hz}), 6.81-6.90(\mathrm{~m}, 4 \mathrm{H}), 6.72(\mathrm{~s}, 1 \mathrm{H}), 6.56(\mathrm{~s}, 1 \mathrm{H}), 4.52(\mathrm{~s}, 4 \mathrm{H})$, 3.75-3.88 (m, 18H), $3.67(\mathrm{t}, 2 \mathrm{H}, J=7.3 \mathrm{~Hz}), 3.45(\mathrm{t}, 2 \mathrm{H}, J=7.3 \mathrm{~Hz}), 2.83-2.97(\mathrm{~m}, 4 \mathrm{H}), 0.24(\mathrm{~s}, 9 \mathrm{H}), 0.22(\mathrm{~s}, 9 \mathrm{H}) ;{ }^{13} \mathrm{C}$ NMR $\left(\mathrm{CDCl}_{3}, 75 \mathrm{MHz}\right): \delta$ 159.77, 159.56, 154.51, 154.28, 148.98, 148.89, 148.68, 130.40, 130.29, 129.82, 129.66, $129.09,128.71,116.05,115.75,114.71,114.47,114.38,113.98,113.90,97.75,96.90,56.59,56.53,55.67,53.31$, $48.18,47.49,44.48,35.27,33.45,31.98,-0.14,-0.28$.

3-(4-Methoxyphenyl)propynoic acid [2-(2-bromo-4,5-dimethoxyphenyl)ethyl]-(4-methoxybenzyl)amide (3d). Synthesized from (4-methoxyphenyl)propynoic acid $194 \mathrm{mg}$ and amine 2a $380 \mathrm{mg}$. Yield: $538 \mathrm{mg}(100 \%) .{ }^{1} \mathrm{H}$ NMR $\left(\mathrm{CDCl}_{3}, 300 \mathrm{MHz}\right): \delta 7.47(\mathrm{~m}, 4 \mathrm{H}), 7.25(\mathrm{~m}, 2 \mathrm{H}), 6.81-7.00(\mathrm{~m}, 10 \mathrm{H}), 6.76(\mathrm{~s}, 1 \mathrm{H}), 6.56(\mathrm{~s}, 1 \mathrm{H}), 4.60(\mathrm{~s}, 4 \mathrm{H}), 3.63-$ $3.93(\mathrm{~m}, 26 \mathrm{H}), 3.52(\mathrm{t}, 2 \mathrm{H}, J=8.0 \mathrm{~Hz}), 2.91-3.02(\mathrm{~m}, 4 \mathrm{H}) ;{ }^{13} \mathbf{C ~ N M R}\left(\mathrm{CDCl}_{3}, 75 \mathrm{MHz}\right): \delta 1^{161.74}, 161.38,159.73$, $159.56,155.44,155.29,148.94,148.87,148.68,134.65,134.52,130.52,130.31,129.98,129.55,129.34,129.91$, $116.02,115.76,114.62,114.55,114.41,114.01,113.82,112.89,112.67,91.14,90.77,81.66,81.37,56.53,56.35$, $55.78,55.68,53.34,48.43,47.81,44.65,36.46,35.64,33.63$.

3-(4-Methoxyphenyl)propynoic acid [2-(2-bromo-4,5-dimethoxyphenyl)ethyl]-methylamide (3e). Synthesized from (4-methoxyphenyl)propynoic acid $194 \mathrm{mg}$ and amine $\mathbf{2 b} 274 \mathrm{mg}$. Yield: $432 \mathrm{mg}(100 \%)$. ${ }^{\mathbf{1}} \mathbf{H} \mathbf{~ N M R}\left(\mathrm{CDCl}_{3}, 400\right.$ $\mathrm{MHz})$ : $\delta$ 7.44-7.50 (m, 4H), 7.00 (s, 1H), $6.94(\mathrm{~s}, 1 \mathrm{H}), 6.84-6.88(\mathrm{~m}, 4 \mathrm{H}), 6.80(\mathrm{~s}, 1 \mathrm{H}), 6.64(\mathrm{~s}, 1 \mathrm{H}), 3.84-3.87(\mathrm{~m}, 6 \mathrm{H})$, $3.82(\mathrm{~s}, 6 \mathrm{H}), 3.78(\mathrm{~s}, 3 \mathrm{H}), 3.74(\mathrm{~s}, 3 \mathrm{H}), 3.69(\mathrm{~s}, 3 \mathrm{H}), 3.65(\mathrm{t}, 2 \mathrm{H}, \mathrm{J}=7.6 \mathrm{~Hz}), 3.15(\mathrm{~s}, 3 \mathrm{H}), 2.94-3.04(\mathrm{~m}, 7 \mathrm{H}) ;{ }^{13} \mathrm{C} \mathrm{NMR}$ $\left(\mathrm{CDCl}_{3}, 75 \mathrm{MHz}\right): \delta$ 161.39, 161.32, 155.24, 148.98, 148.92, 148.70, 134.52, 130.31, 129.74, 116.09, 115.82, 114.60, $114.51,114.38,113.95,113.86,112.89,112.75,90.35,81.45,81.15,56.53,56.41,55.77,51.58,47.40,37.67,35.58$, 35.40, 33.50, 33.20.

3-Phenylpropynoic acid [2-(2-bromo-4,5-dimethoxyphenyl)ethyl]methylamide (3f). Synthesized from phenylpropynoic acid $161 \mathrm{mg}$ and amine $2 \mathrm{~b} 274 \mathrm{mg}$. Yield: $402 \mathrm{mg}(100 \%) .{ }^{1} \mathbf{H}$ NMR $\left(\mathrm{CDCl}_{3}, 300 \mathrm{MHz}\right): \delta 7.48-7.57$ $(\mathrm{m}, 4 \mathrm{H}), 7.31-7.44(\mathrm{~m}, 6 \mathrm{H}), 7.00(\mathrm{~s}, 1 \mathrm{H}), 6.92(\mathrm{~s}, 1 \mathrm{H}), 6.80(\mathrm{~s}, 1 \mathrm{H}), 6.62(\mathrm{~s}, 1 \mathrm{H}), 3.82-3.91(\mathrm{~m}, 8 \mathrm{H}), 3.77(\mathrm{~s}, 3 \mathrm{H}), 3.6-$ $3.74(\mathrm{~m}, 5 \mathrm{H}), 3.16(\mathrm{~s}, 3 \mathrm{H}), 2.94-3.08(\mathrm{~m}, 7 \mathrm{H}) ;{ }^{13} \mathrm{C}$ NMR $\left(\mathrm{CDCl}_{3}, 75 \mathrm{MHz}\right): \delta$ 154.93, 148.96, 148.74, 132.87, 132.73 , $130.44,130.34,130.22,129.64,128.92,128.85,121.02,120.90,116.09,15.84,114.68,114.41,113.93,113.80$, $90.72,89.79,82.15,81.90,56.52,56.35,53.85,51.63,47.76,37.67,35.40,33.48,33.26$.

2-Butynoic acid [2-(2-bromo-4,5-dimethoxyphenyl)ethyl]methylamide (3g). Synthesized from 2-butynoic acid 92 $\mathrm{mg}$ and amine 2b $274 \mathrm{mg}$. Yield: $332 \mathrm{mg}(98 \%)$. ${ }^{1} \mathbf{H}$ NMR $\left(\mathrm{CDCl}_{3}, 300 \mathrm{MHz}\right): \delta 6.98-7.02(\mathrm{~m}, 2 \mathrm{H}), 6.76(\mathrm{~s}, 1 \mathrm{H}), 6.64$ (s, 1H), $3.85(\mathrm{~s}, 12 \mathrm{H}), 3.77(\mathrm{t}, 2 \mathrm{H}, J=7.1 \mathrm{~Hz}), 3.59(\mathrm{t}, 2 \mathrm{H}, J=7.3 \mathrm{~Hz}), 3.06(\mathrm{~s}, 3 \mathrm{H}), 2.88-2.99(\mathrm{~m}, 7 \mathrm{H}), 2.00(\mathrm{~s}, 3 \mathrm{H})$, $1.96(\mathrm{~s}, 3 \mathrm{H}) ;{ }^{13} \mathrm{C}$ NMR $\left(\mathrm{CDCl}_{3}, 75 \mathrm{MHz}\right): \delta 155.04,148.96,148.90,148.65,130.26,130.16,129.74,116.02,115.78$, $114.75,114.36,113.95,113.83,89.46,88.61,73.96,73.56,56.59,56.53,51.30,49.29,47.18,37.53,35.27,34.33$, $33.39,33.04,26.04,25.34$. 
3-Trimethylsilylpropynoic acid [2-(2-bromo-4,5-dimethoxyphenyl)ethyl]methyl amide (3h). Synthesized from trimethylsilylpropynoic acid $156 \mathrm{mg}$ and amine $2 \mathrm{~b} 274 \mathrm{mg}$. Yield: $386 \mathrm{mg}(97 \%)$. ${ }^{1} \mathrm{H}$ NMR $\left(\mathrm{CDCl}_{3}, 300 \mathrm{MHz}\right): \delta 7.01$ $(\mathrm{s}, 1 \mathrm{H}), 6.99(\mathrm{~s}, 1 \mathrm{H}), 6.75(\mathrm{~s}, 1 \mathrm{H}), 6.62(\mathrm{~s}, 1 \mathrm{H}), 3.75-3.90(\mathrm{~m}, 14 \mathrm{H}), 3.59(\mathrm{t}, 2 \mathrm{H}, J=7.8 \mathrm{~Hz}), 3.08(\mathrm{~s}, 3 \mathrm{H}), 2.89-3.00$ $(\mathrm{m}, 7 \mathrm{H}), 0.2-0.25(\mathrm{~m}, 18 \mathrm{H}) ;{ }^{13} \mathrm{C}$ NMR $\left(\mathrm{CDCl}_{3}, 75 \mathrm{MHz}\right): \delta 154.30,149.01,148.93,148.72,130.18,129.58,116.09$, $115.81,114.71,114.35,113.89,96.84,96.23,56.54,51.42,47.27,37.64,35.15,33.32,33.02,-0.17,-0.24$.

3-Phenylpropynoic acid [2-(2-bromo-4,5-dimethoxyphenyl)ethyl]amide (3i). (one rotamer almost exclusively) Synthesized from phenylpropynoic acid $161 \mathrm{mg}$ and amine $2 \mathrm{c} 260 \mathrm{mg}$. Yield: $369 \mathrm{mg}(95 \%)$. ${ }^{1} \mathbf{H}$ NMR $\left(\mathrm{CDCl}_{3}, 400\right.$ $\mathrm{MHz}): \delta 7.51(\mathrm{~d}, 2 \mathrm{H}, J=7.6 \mathrm{~Hz}), 7.32-7.43(\mathrm{~m}, 3 \mathrm{H}), 7.02(\mathrm{~s}, 1 \mathrm{H}), 6.76(\mathrm{~s}, 1 \mathrm{H}), 6.01(\mathrm{bs}, 1 \mathrm{H}), 3.86(\mathrm{~s}, 6 \mathrm{H}), 3.55-3.64$ $(\mathrm{m}, 2 \mathrm{H}), 2.96(\mathrm{t}, 2 \mathrm{H}, J=7.1 \mathrm{~Hz}) ;{ }^{13} \mathrm{C}$ NMR $\left(\mathrm{CDCl}_{3}, 75 \mathrm{MHz}\right): \delta 153.95,148.90,148.77,132.88,130.50,130.10$, $128.94,120.51,115.99,114.56,113.63,85.17,83.37,56.56,56.51,40.21,35.54$.

2-Butynoic acid [2-(2-bromo-4,5-dimethoxyphenyl)ethyl]amide (3j). (one rotamer almost exclusively) Synthesized from 2-butynoic acid $92 \mathrm{mg}$ and amine $2 \mathrm{c} 260 \mathrm{mg}$. Yield: $268 \mathrm{mg}(82 \%)$. ${ }^{1} \mathrm{H}$ NMR $\left(\mathrm{CDCl}_{3}, 300 \mathrm{MHz}\right): \delta 7.00(\mathrm{~s}, 1 \mathrm{H})$, $6.73(\mathrm{~s}, 1 \mathrm{H}), 5.90(\mathrm{bs}, 1 \mathrm{H}), 3.85(\mathrm{~s}, 6 \mathrm{H}), 3.46-3.58(\mathrm{~m}, 2 \mathrm{H}), 2.91(\mathrm{t}, 2 \mathrm{H}, \mathrm{J}=6.9 \mathrm{~Hz}), 1.92(\mathrm{~s}, 3 \mathrm{H}) ;{ }^{13} \mathrm{C} \mathrm{NMR}\left(\mathrm{CDCl}_{3}, 75\right.$ $\mathrm{MHz}): \delta$ 153.98, 148.86, 148.74, 130.14, 115.97, 114.53, 113.83, 83.71, 75.24, 56.56, 56.51, 39.99, 35.48, 4.02.

2-Octynoic acid [2-(2-bromophenyl)ethyl]methylamide (3k). Synthesized from 2-octynoic acid $154 \mathrm{mg}$ and amine 2d $214 \mathrm{mg}$. Yield: $327 \mathrm{mg}(97 \%)$. ${ }^{1} \mathrm{H}$ NMR $\left(\mathrm{CDCl}_{3}, 300 \mathrm{MHz}\right): \delta 7.50-7.59(\mathrm{~m}, 2 \mathrm{H}), 7.06-7.30(\mathrm{~m}, 6 \mathrm{H}), 3.80(\mathrm{t}, 2 \mathrm{H}, J=$ 7.5 Hz), 3.52-3.67 (m, 2H), 2.94-3.12 (m, 10H), 2.24-2.42 (m, 4H), 1.48-1.65 (m, 4H), 1.24-1.47 (m, 8H), 0.83-0.97 $(\mathrm{m}, 6 \mathrm{H}) ;{ }^{13} \mathrm{C}$ NMR $\left(\mathrm{CDCl}_{3}, 75 \mathrm{MHz}\right): \delta 155.15,138.52,137.90,133.33,133.21,131.62,131.47,128.94,128.70$, $128.15,128.09,124.92,124.80,93.68,92.99,74.66,74.11,51.20,47.19,37.44,35.61,33.95,32.99,31.52,31.41$, $27.85,26.01,22.53,19.37,19.28,14.29$.

3-(4-Methoxyphenyl)propynoic acid [2-(2-bromo-phenyl)ethyl]methylamide (3I). (mixture of rotamers $2 / 3$ ) Synthesized from 3-(4-methoxyphenyl)propynoic acid $194 \mathrm{mg}$ and amine 2d $214 \mathrm{mg}$. Yield: $361 \mathrm{mg}(96 \%)$. ${ }^{1} \mathbf{H}$ NMR $\left(\mathrm{CDCl}_{3}, 300 \mathrm{MHz}\right): \delta$ 7.43-7.59 (m, 6H), 7.05-7.16 (m, 4H), 7.04-7.14 (m, 2H), $6.87(\mathrm{~d}, 4 \mathrm{H}, J=8.5 \mathrm{~Hz}), 3.79-3.92(\mathrm{~m}$, $8 \mathrm{H}), 3.60-3.71(\mathrm{~m}, 2 \mathrm{H}), 3.17(\mathrm{~s}, 2 \mathrm{H}), 3.00-3.13(\mathrm{~m}, 8 \mathrm{H}) ;{ }^{13} \mathrm{C} \mathrm{NMR}\left(\mathrm{CDCl}_{3}, 75 \mathrm{MHz}\right): \delta 161.38,161.33,155.26$, 137.87, 134.67, 134.59, 133.39, 133.24, 131.65, 131.53, 129.00, 128.74, 128.21, 128.15, 124.92, 124.83, 114.59, 114.54, 112.68, 91.23, 90.50, 81.48, 81.09, 55.77, 51.36, 47.33, 37.53, 36.03, 35.71, 34.03, 33.14.

2-Butynoic acid [2-(2-bromophenyl)ethyl]methylamide $(3 \mathrm{~m})$. (mixture of rotamers $1 / 2)$ Synthesized from 2butynoic acid $92 \mathrm{mg}$ and amine 2d $214 \mathrm{mg}$. Yield: $236 \mathrm{mg}(84 \%)$. ${ }^{1} \mathbf{H}$ NMR $\left(\mathrm{CDCl}_{3}, 300 \mathrm{MHz}\right): \delta 7.49-7.57(\mathrm{~m}, 1 \mathrm{H})$, 7.20-7.29 (m, 1H), 7.05-7.18 (m, 2H), 3.74-3.86 (m, 1H), 3.51-3.66 (m, 1H), 2.91-3.11 (m, 5H), $2.00(\mathrm{~s}, 1 \mathrm{H}), 1.94(\mathrm{~s}$, $2 \mathrm{H}) ;{ }^{13} \mathrm{C}$ NMR $\left(\mathrm{CDCl}_{3}, 75 \mathrm{MHz}\right): \delta 155.11,155.05,138.46,137.81,133.36,133.26,133.22,131.60,131.38,128.97$, 128.73, 128.18, 128.12, 124.98, 124.80, 89.50, 88.77, 73.93, 73.40, 51.08, 37.47, 35.57, 33.93, 32.95, 4.53, 4.52.

3-(4-Methoxyphenyl)propynoic acid [2-(2-bromophenyl)ethyl]isopropylamide (3n). Synthesized from 3-(4methoxyphenyl)propynoic acid $194 \mathrm{mg}$ and amine $2 \mathrm{e} 242 \mathrm{mg}$. Yield: $360 \mathrm{mg}(90 \%)$. ${ }^{1} \mathrm{H}$ NMR $\left(\mathrm{CDCl}_{3}, 300 \mathrm{MHz}\right): \delta$ 7.46-7.59 (m, 6H), 7.19-7.43 (m, 4H), 7.04-7.17 (m, 2H), $6.91(\mathrm{~d}, 4 \mathrm{H}, J=8.7 \mathrm{~Hz}), 4.76(\mathrm{~m}, 2 \mathrm{H}), 3.72-3.96(\mathrm{~m}, 8 \mathrm{H})$, $3.48(\mathrm{t}, 2 \mathrm{H}, J=8.0 \mathrm{~Hz}), 3.04-3.24(\mathrm{~m}, 4 \mathrm{H}), 1.17-1.41(\mathrm{~m}, 12 \mathrm{H}) ;{ }^{3} \mathrm{C} \mathrm{NMR}\left(\mathrm{CDCl}_{3}, 75 \mathrm{MHz}\right): \delta$ 161.32, 155.38, 155.14, 139.22, 138.60, 134.54, 133.36, 133.05, 131.94, 131.45, 128.88, 128.60, 128.21, 128.13, 124.80, 124.74, 114.60, 112.93, 90.65, 90.13, 82.40, 81.59, 55.77, 51.05, 46.11, 45.13, 41.34, 38.59, 35.39, 21.59, 20.87.

3-(3,4,5-Trimethoxyphenyl)propynoic acid [2-(2-bromophenyl)ethyl]isopropylamide (30). Synthesized from 3(3,4,5-trimethoxyphenyl)propynoic acid $260 \mathrm{mg}$ and amine $2 \mathrm{e} 242 \mathrm{mg}$. Yield: $331 \mathrm{mg}(72 \%)$. ${ }^{1} \mathbf{H} \mathbf{~ N M R}\left(\mathrm{CDCl}_{3}, 300\right.$ $\mathrm{MHz}): \delta 7.56(\mathrm{~d}, 2 \mathrm{H}, J=8.3 \mathrm{~Hz}), 7.18-7.43(\mathrm{~m}, 4 \mathrm{H}), 7.06-7.17(\mathrm{~m}, 2 \mathrm{H}), 6.81(\mathrm{~s}, 4 \mathrm{H}), 4.75(\mathrm{~m}, 2 \mathrm{H}), 3.72-3.97(\mathrm{~m}$, 20H), 3.44-3.55 (m, 2H), 3.07-3.25 (m, 4H), 1.22-1.36 (m, 12H); ${ }^{13} \mathbf{C ~ N M R ~}\left(\mathrm{CDCl}_{3}, 75 \mathrm{MHz}\right)$ : $\delta$ 155.08, 154.80, $153.50,140.55,139.10,138.54,133.39,133.03,131.90,131.29,128.92,128.61,128.11,124.77,115.87,115.82$, $110.08,110.03,90.47,89.80,82.27,81.48,61.34,56.57,51.113,46.20,45.09,41.36,38.55,35.30,21.61,20.85$.

2-Butynoic acid [3-(2-bromo-4,5-dimethoxyphenyl)propyl]-(4-methoxybenzyl)amide (10a). Synthesized from 2butynoic acid $92 \mathrm{mg}$ and [3-(2-Bromo-4,5-dimethoxyphenyl)propyl]-(4-methoxybenzyl)amine $408 \mathrm{mg}$. Yield: $440 \mathrm{mg}$ (96\%). ${ }^{1} \mathrm{H}$ NMR $\left(\mathrm{CDCl}_{3}, 400 \mathrm{MHz}\right): \delta 7.17(\mathrm{~d}, 4 \mathrm{H}, J=8.7 \mathrm{~Hz}), 6.99(\mathrm{~d}, 2 \mathrm{H}, J=7.7 \mathrm{~Hz}), 6.81-6.92(\mathrm{~m}, 4 \mathrm{H}), 6.72(\mathrm{~s}$, $1 \mathrm{H}), 6.61(\mathrm{~s}, 1 \mathrm{H}), 4.72(\mathrm{~s}, 2 \mathrm{H}), 4.57(\mathrm{~s}, 2 \mathrm{H}), 3.78-3.9(\mathrm{~m}, 18 \mathrm{H}), 3.51(\mathrm{t}, 2 \mathrm{H}, J=7.1 \mathrm{~Hz}), 3.52(\mathrm{t}, 2 \mathrm{H}, J=7.1 \mathrm{~Hz}), 2.55-$ $2.72(\mathrm{~m}, 4 \mathrm{H}), 2.02(\mathrm{~s}, 3 \mathrm{H}), 1.97(\mathrm{~s}, 3 \mathrm{H}), 1.72-1.92(\mathrm{~m}, 4 \mathrm{H}) ;{ }^{13} \mathrm{C}$ NMR $\left(\mathrm{CDCl}_{3}, 75 \mathrm{MHz}\right): \delta$ 159.68, 159.44, 155.32 , $155.14,148.78,148.41,148.23,133.09,132.81,130.01,129.34,128.85,116.03,115.91,114.53,114.38,114.23$, 113.31, 89.56, 89.49, 74.08, 73.87, 56.56, 56.49, 55.67, 52.37, 47.82, 47.10, 43.49, 33.42, 29.15, 27.43, 4.51, 4.37.

3-Phenylpropynoic acid [3-(2-bromo-4,5-dimethoxyphenyl)propyl]-(4-methoxy-benzyl)amide (10b). Synthesized from phenylpropynoic acid $161 \mathrm{mg}$ and [3-(2-Bromo-4,5-dimethoxyphenyl)propyl]-(4-methoxybenzyl)amine $408 \mathrm{mg}$. Yield: $514 \mathrm{mg}(98 \%) .{ }^{1} \mathrm{H}$ NMR $\left(\mathrm{CDCl}_{3}, 300 \mathrm{MHz}\right): \delta$ 7.47-7.57 (m, 4H), 7.31-7.46 (m, 6H), 7.18-7.30 (m, 4H), 6.83$7.02(\mathrm{~m}, 6 \mathrm{H}), 6.75(\mathrm{~s}, 1 \mathrm{H}), 6.59(\mathrm{~s}, 1 \mathrm{H}), 4.82(\mathrm{~s}, 2 \mathrm{H}), 4.65(\mathrm{~s}, 2 \mathrm{H}), 3.73-3.93(\mathrm{~m}, 18 \mathrm{H}), 3.64(\mathrm{t}, 2 \mathrm{H}, \mathrm{J}=7.2 \mathrm{~Hz}), 3.43(\mathrm{t}$, $2 \mathrm{H}, J=7.2 \mathrm{~Hz}), 2.60-2.74(\mathrm{~m}, 4 \mathrm{H}), 1.75-1.98(\mathrm{~m}, 4 \mathrm{H}) ;{ }^{13} \mathrm{C} \mathrm{NMR}\left(\mathrm{CDCl}_{3}, 75 \mathrm{MHz}\right): \delta 159.72,159.49,155.14,154.96$, $148.75,148.37,148.22,132.96,132.78,132.72,132.62,130.48,130.09,129.43,129.22,128.92,128.71,120.83$, 
$115.87,114.59,114.41,114.32,114.26,113.26,113.19,90.63,82.40,82.15,56.45,56.38,55.64,52.61,48.22$, 47.40, 43.63, 33.54, 33.44, 29.36, 27.53.

\section{General procedure for the Heck reductive cyclization:}

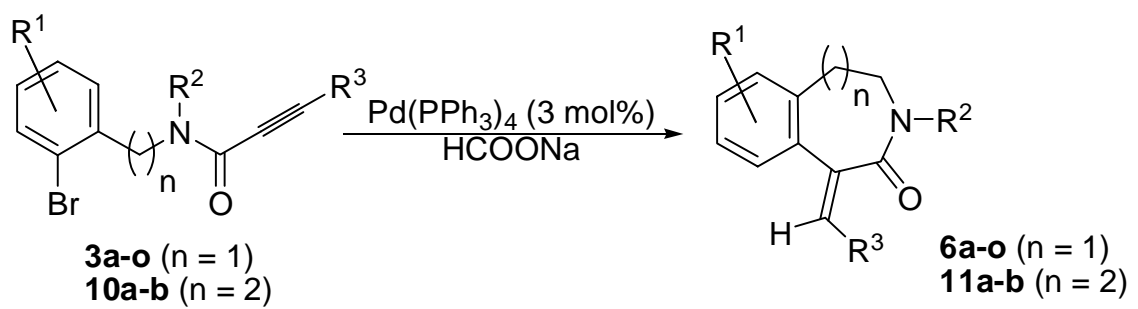

$\mathrm{Pd}\left(\mathrm{PPh}_{3}\right)_{4} 14 \mathrm{mg}$ ( $\left.3 \mathrm{~mol} \%\right)$ and HCOONa $41 \mathrm{mg}$ (1.5 equiv) were loaded into the microwave instrument vial. The vial was evacuated and flushed with argon. Propynoic acid amide (3 or 10) (0.4 mmol) dissolved in DMF (4.5 mL) was added, followed by distilled water $(1.5 \mathrm{~mL})$. The vial was sealed and irradiated with stirring at a ceiling temperature of $110^{\circ} \mathrm{C}$ at $150 \mathrm{~W}$ maximum power level for $15 \mathrm{~min}$. Upon completion of the reaction time the vial was cooled with a stream of air. After dilution with DCM the organic phase was washed several times with brine, dried $\left(\mathrm{MgSO}_{4}\right)$ and concentrated under reduced pressure. Products were isolated by column chromatography on silica gel.

In ${ }^{1} \mathbf{H}$ NMR spectra of benzazepinones 6 , recorded at $25^{\circ} \mathrm{C}$, signals of protons at $\mathrm{C}^{3}$ and $\mathrm{C}^{16}$ (on the example of $6 \mathbf{a}$ ) are often broadened, even merging with the baseline due to the 7-membered ring flipping ${ }^{5}$. Assignment for the $6 \mathbf{a}$ was performed on the bases of COSY, HMBC and HMQC spectra. Configuration of the double bond was established with NOE effect (interaction between protons at $C^{11}$ and $C^{7}$ ).

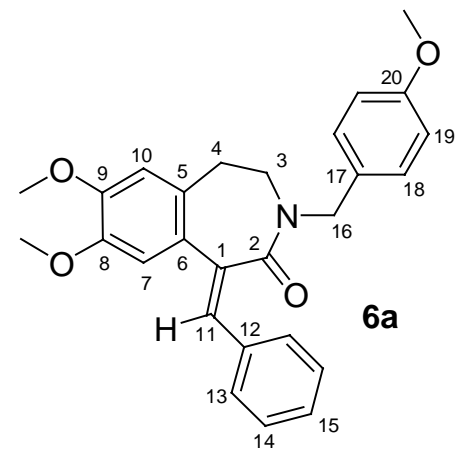

1-Benzylidene-7,8-dimethoxy-3-(4-methoxybenzyl)-1,3,4,5-tetrahydrobenzo[d]azepin-2-one (6a). Synthesized from amide 3a $203 \mathrm{mg}$. Eluent DCM / $\mathrm{Et}_{2} \mathrm{O}(4 / 1)\left(R_{f} 0.46\right)$. Yield: $139 \mathrm{mg}(81 \%)$. ${ }^{1} \mathbf{H} \mathbf{~ N M R}\left(\mathrm{CDCl}_{3}, 400 \mathrm{MHz}: \mathrm{\delta}^{2} .53\right.$ $\left(\mathrm{d}, 2 \mathrm{H}-\mathrm{C}^{13}, J=7.2 \mathrm{~Hz}\right), 7.31\left(\mathrm{~m}, 2 \mathrm{H}-\mathrm{C}^{14}\right), 7.20-7.27\left(\mathrm{~m}, \mathrm{H}-\mathrm{C}^{15}, 2 \mathrm{H}-\mathrm{C}^{18}\right), 6.94\left(\mathrm{~s}, \mathrm{H}-\mathrm{C}^{7}\right), 6.85\left(\mathrm{~d}, 2 \mathrm{H}-\mathrm{C}^{19}, J=8.6 \mathrm{~Hz}\right)$, $6.63\left(\mathrm{~s}, \mathrm{H}-\mathrm{C}^{11}\right), 6.53\left(\mathrm{~s}, \mathrm{H}-\mathrm{C}^{10}\right), 3.91(\mathrm{~s}, 3 \mathrm{H}), 3.84(\mathrm{~s}, 3 \mathrm{H}), 3.79(\mathrm{~s}, 3 \mathrm{H}), 2.90\left(\mathrm{bs}, 2 \mathrm{H}-\mathrm{C}^{4}\right) ;{ }^{13} \mathrm{C} \mathrm{NMR}\left(\mathrm{CDCl} \mathrm{N}_{3}, 100 \mathrm{MHz}\right)$ : ठ $170.67\left(C^{2}\right), 159.21\left(C^{20}\right), 149.12\left(C^{9}\right), 147.81\left(C^{8}\right), 138.70\left(C^{1}\right), 135.68\left(C^{12}\right), 131.28\left(C^{11}\right), 129.75\left(C^{18}\right), 129.42$ $\left(C^{17}\right), 128.62\left(C^{13}\right), 128.46\left(C^{14}\right), 128.21\left(C^{5}\right), 127.97\left(C^{15}\right), 127.64\left(C^{6}\right), 114.09\left(C^{19}\right), 112.85\left(C^{10}\right), 112.50\left(C^{3}\right), 56.10$ $\left(\mathrm{OCH}_{3}\right), 56.00\left(\mathrm{OCH}_{3}\right), 55.29\left(\mathrm{OCH}_{3}\right), 47.55\left(\mathrm{C}^{16}\right), 44.60\left(\mathrm{C}^{3}\right), 31.80\left(\mathrm{C}^{4}\right)$; HRMS calcd. For $\mathrm{C}_{27} \mathrm{H}_{27} \mathrm{NO}_{4}: \mathrm{m}_{2} \mathrm{z} 429.1940$, found $\mathrm{m} / \mathrm{z} 429.1937$

1-Ethylidene-7,8-dimethoxy-3-(4-methoxybenzyl)-1,3,4,5-tetrahydrobenzo[d]azepin-2-one (6b). Synthesized from amide 3b $179 \mathrm{mg}$. Eluent DCM / $\mathrm{Et}_{2} \mathrm{O}(4 / 1)\left(R_{f} 0.34\right)$. Yield: $96 \mathrm{mg}(65 \%)$. ${ }^{1} \mathbf{H} \mathbf{N M R}\left(\mathrm{CDCl}_{3}, 300 \mathrm{MHz}\right): \delta 7.23$ $(\mathrm{d}, 2 \mathrm{H}, J=8.6 \mathrm{~Hz}), 6.86(\mathrm{~d}, 2 \mathrm{H}, J=8.6 \mathrm{~Hz}), 6.77(\mathrm{~s}, 1 \mathrm{H}), 6.49(\mathrm{~s}, 1 \mathrm{H}), 5.93(\mathrm{q}, 1 \mathrm{H}, J=7.0 \mathrm{~Hz}), 4.63(\mathrm{bs}, 2 \mathrm{H}), 3.87(\mathrm{~s}$, $3 \mathrm{H}), 3.82(\mathrm{~s}, 3 \mathrm{H}), 3.79(\mathrm{~s}, 3 \mathrm{H}), 3.58(\mathrm{bs}, 2 \mathrm{H}), 2.84(\mathrm{t}, 2 \mathrm{H}, \mathrm{J}=5.4 \mathrm{~Hz}), 1.97$ (d, 3H, J = $7.0 \mathrm{~Hz}) ;{ }^{13} \mathrm{C} \mathrm{NMR}\left(\mathrm{CDCl} \mathrm{N}_{3}, 75\right.$ $\mathrm{MHz}$ ) : $\delta 171.10,159.43,148.89,147.80,139.91,130.01,129.84,127.91,127.42,144.44,112.98,112.78,56.30$, 55.68, 48.10, 44.97, 32.53, 16.02; HRMS calcd. For $\mathrm{C}_{22} \mathrm{H}_{25} \mathrm{NO}_{4}: \mathrm{m} / \mathrm{z} 367.1784$, found m/z 367.1793

7,8-Dimethoxy-3-(4-methoxy-benzyl)-1-trimethylsilylmethylene-1,3,4,5-tetrahydrobenzo[d]azepin-2-one (6c). Synthesized from amide 3c $202 \mathrm{mg}$ and HCOONa $82 \mathrm{mg}$ (3 equiv). Solvent - mixture of DME (4.8 mL) and $\mathrm{H}_{2} \mathrm{O}(1.2$ $\mathrm{mL})$. Reaction temperature $120^{\circ} \mathrm{C}$. Eluent DCM / $\mathrm{Et}_{2} \mathrm{O}(20 / 1)\left(R_{f} 0.36\right)$. Yield: $61 \mathrm{mg}(36 \%)$. ${ }^{1} \mathbf{H ~ N M R}\left(\mathrm{CDCl} \mathbf{N}_{3}, 400\right.$ $\mathrm{MHz}): \delta 7.23(\mathrm{~d}, 2 \mathrm{H}, J=8.8 \mathrm{~Hz}), 6.83(\mathrm{~d}, 2 \mathrm{H}, J=8.8 \mathrm{~Hz}), 6.79(\mathrm{~s}, 1 \mathrm{H}), 6.42(\mathrm{~s}, 1 \mathrm{H}), 6.02(\mathrm{~s}, 1 \mathrm{H}), 4.59(\mathrm{~s}, 2 \mathrm{H}), 3.88$ $(\mathrm{s}, 3 \mathrm{H}), 3.80(\mathrm{~s}, 3 \mathrm{H}), 3.78(\mathrm{~s}, 3 \mathrm{H}), 3.61(\mathrm{~m}, 2 \mathrm{H}), 2.75(\mathrm{~m}, 2 \mathrm{H}), 0.18(\mathrm{~s}, 9 \mathrm{H}) ;{ }^{13} \mathbf{C ~ N M R}\left(\mathrm{CDCl}_{3}, 100 \mathrm{MHz}\right): \delta 171.65$, 159.13, 154.33, 149.00, 147.62, 135.95, 130.00, 129.88, 129.48, 126.90, 114.01, 112.83, 112.23, 56.05, 55.89, 55.25, 48.26, 44.68, 32.33, -0.59; HRMS calcd. For $\mathrm{C}_{24} \mathrm{H}_{31} \mathrm{NO}_{4} \mathrm{Si}: \mathrm{m} / \mathrm{z} 425.2022$, found m/z 425.2019 
7,8-Dimethoxy-3-(4-methoxybenzyl)-1-(4-methoxybenzylidene)-1,3,4,5-tetrahydrobenzo[d]azepin-2-one (6d). Synthesized from amide 3d $215 \mathrm{mg}$. Eluent DCM / $\mathrm{Et}_{2} \mathrm{O}(4 / 1)\left(R_{f} 0.47\right)$. Yield: $142 \mathrm{mg}(77 \%) .{ }^{1} \mathbf{H} \mathbf{~ N M R}\left(\mathrm{CDCl}_{3}, 400\right.$ $\mathrm{MHz}): \delta 7.49(\mathrm{~d}, 2 \mathrm{H}, J=8.7 \mathrm{~Hz}), 7.25(\mathrm{~d}, 2 \mathrm{H}, J=8.5 \mathrm{~Hz}), 6.93(\mathrm{~s}, 1 \mathrm{H}), 6.84-6.87(\mathrm{~m}, 4 \mathrm{H}), 6.57(\mathrm{~s}, 1 \mathrm{H}), 6.52(\mathrm{~s}, 1 \mathrm{H})$, $3.91(\mathrm{~s}, 3 \mathrm{H}), 3.84(\mathrm{~s}, 3 \mathrm{H}), 3.82(\mathrm{~s}, 3 \mathrm{H}), 3.80(\mathrm{~s}, 3 \mathrm{H}), 2.89(\mathrm{bs}, 2 \mathrm{H}) ;{ }^{13} \mathrm{C} \mathbf{N M R}\left(\mathrm{CDCl}_{3}, 75 \mathrm{MHz}\right): \delta 171.44,159.83$, $159.53,149.20,148.02,136.63,131.38,130.49,130.13,129.80,128.76,127.79,114.44,114.32,112.98,112.70$, 56.44, 56.35, 55.69, 47.91, 44.99, 32.16; HRMS calcd. For $\mathrm{C}_{28} \mathrm{H}_{29} \mathrm{NO}_{5}: \mathrm{m} / \mathrm{z} 459.2046$, found $\mathrm{m} / \mathrm{z} 459.2040$

7,8-Dimethoxy-1-(4-methoxybenzylidene)-3-methyl-1,3,4,5-tetrahydrobenzo[d]azepin-2-one (6e). Synthesized from amide $3 \mathrm{e} 173 \mathrm{mg}$. Eluent DCM / $\mathrm{Et}_{2} \mathrm{O}(4 / 1)\left(R_{f} 0.32\right)$. Yield: $100 \mathrm{mg}(71 \%)$. ' $\mathrm{H} \mathbf{N M R}\left(\mathrm{CDCl}_{3}, 400 \mathrm{MHz}\right): \delta 7.48$ $(\mathrm{d}, 2 \mathrm{H}, J=8.6 \mathrm{~Hz}), 6.94(\mathrm{~s}, 1 \mathrm{H}), 6.86(\mathrm{~d}, 2 \mathrm{H}, J=8.6 \mathrm{~Hz}), 6.58(\mathrm{~s}, 1 \mathrm{H}), 6.55(\mathrm{~s}, 1 \mathrm{H}), 3.89(\mathrm{~s}, 3 \mathrm{H}), 3.85(\mathrm{~s}, 3 \mathrm{H}), 3.80(\mathrm{~s}$, 3H), 3.14 (s, 3H), 3.04 (bs, 2H); ${ }^{13} \mathbf{C}$ NMR $\left(\mathrm{CDCl}_{3}, 75 \mathrm{MHz}\right): \delta 171.65,159.82,149.18,148.04,136.41,131.10$, 130.43, 128.81, 128.64, 127.73, 114.60, 114.40, 113.01, 112.74, 56.44, 56.36, 55.66, 48.06, 32.96, 31.53; HRMS calcd. For $\mathrm{C}_{21} \mathrm{H}_{23} \mathrm{NO}_{4}: \mathrm{m} / \mathrm{z} 353.1627$, found $\mathrm{m} / \mathrm{z} 353.1631$

1-Benzylidene-7,8-dimethoxy-3-methyl-1,3,4,5-tetrahydrobenzo[d]azepin-2-one (6f). Synthesized from amide $3 f$ $161 \mathrm{mg}$. Eluent DCM / Et $2 \mathrm{O}(4 / 1)\left(R_{f} 0.43\right)$. Yield: $88 \mathrm{mg}(68 \%)$. ${ }^{1} \mathrm{H}$ NMR $\left(\mathrm{CDCl}_{3}, 300 \mathrm{MHz}\right): \delta 7.52(\mathrm{~d}, 2 \mathrm{H}, J=7.4$ $\mathrm{Hz}), 7.22-7.35(\mathrm{~m}, 3 \mathrm{H}), 6.92(\mathrm{~s}, 1 \mathrm{H}), 6.61(\mathrm{~s}, 1 \mathrm{H}), 6.59(\mathrm{~s}, 1 \mathrm{H}), 3.89(\mathrm{~s}, 3 \mathrm{H}), 3.85(\mathrm{~s}, 3 \mathrm{H}), 3.13(\mathrm{~s}, 3 \mathrm{H}), 3.04(\mathrm{bs}, 2 \mathrm{H})$; ${ }^{13} \mathrm{C}$ NMR $\left(\mathrm{CDCl}_{3}, 75 \mathrm{MHz}\right): \delta 171.35,149.38,148.07,138.75,135.89,131.39,128.96,128.52,128.40,127.94$, $113.07,112.74,56.44,56.38,48.02,32.93,31.50$; HRMS calcd. For $\mathrm{C}_{20} \mathrm{H}_{21} \mathrm{NO}_{3}: \mathrm{m} / \mathrm{z} 323.1521$, found $\mathrm{m} / \mathrm{z} 323.1515$

1-Ethylidene-7,8-dimethoxy-3-methyl-1,3,4,5-tetrahydrobenzo[d]azepin-2-one (6g). Synthesized from amide $\mathbf{3 g}$ 136 mg. Eluent DCM / Et $\mathrm{t}_{2} \mathrm{O}(4 / 1)\left(R_{f} 0.33\right)$. Yield: $64 \mathrm{mg}(61 \%) .{ }^{1} \mathrm{H}$ NMR $\left(\mathrm{CDCl}_{3}, 300 \mathrm{MHz}\right): \delta 6.75(\mathrm{~s}, 1 \mathrm{H}), 6.65(\mathrm{~s}$, $1 \mathrm{H}), 5.91(\mathrm{q}, 1 \mathrm{H}, J=6.7 \mathrm{~Hz}), 3.86(\mathrm{~s}, 3 \mathrm{H}), 3.84(\mathrm{~s}, 3 \mathrm{H}), 3.68(\mathrm{bs}, 2 \mathrm{H}), 3.09(\mathrm{~s}, 3 \mathrm{H}), 3.00(\mathrm{~m}, 2 \mathrm{H}), 1.92(\mathrm{~d}, 3 \mathrm{H}, J=6.7$ $\mathrm{Hz}) ;{ }^{13} \mathrm{C}$ NMR $\left(\mathrm{CDCl}_{3}, 75 \mathrm{MHz}\right): \delta 171.20,148.89,147.80,139.84,129.63,127.97,127.33,112.98,112.83,56.29$, 48.03, 33.11, 31.87, 15.90; HRMS calcd. For $\mathrm{C}_{15} \mathrm{H}_{19} \mathrm{NO}_{3}: \mathrm{m} / \mathrm{z} 261.1365$, found $\mathrm{m} / \mathrm{z} 261.1371$

7,8-Dimethoxy-3-methyl-1-trimethylsilylmethylene-1,3,4,5-tetrahydrobenzo[d]azepin-2-one (6h). Synthesized from amide $3 \mathrm{~h} 159 \mathrm{mg}$ and HCOONa $82 \mathrm{mg}$ (3 equiv). Solvent - mixture of DME $(4.8 \mathrm{~mL})$ and $\mathrm{H}_{2} \mathrm{O}(1.2 \mathrm{~mL})$. Reaction temperature $120^{\circ} \mathrm{C}$. Eluent DCM / $\mathrm{Et}_{2} \mathrm{O}(4 / 1)\left(R_{f} 0.47\right)$. Yield: $41 \mathrm{mg}(32 \%)$. ${ }^{1} \mathrm{H} \mathrm{NMR}\left(\mathrm{CDCl}_{3}, 400 \mathrm{MHz}\right): \delta$ $6.77(\mathrm{~s}, 1 \mathrm{H}), 6.50(\mathrm{~s}, 1 \mathrm{H}), 5.99(\mathrm{~s}, 1 \mathrm{H}), 3.85(\mathrm{~s}, 3 \mathrm{H}), 3.81(\mathrm{~s}, 3 \mathrm{H}), 3.65(\mathrm{~m}, 2 \mathrm{H}), 3.03(\mathrm{~s}, 3 \mathrm{H}), 2.99(\mathrm{~m}, 2 \mathrm{H}), 0.15(\mathrm{~s}$, 9H); ${ }^{3} \mathrm{C}$ NMR $\left(\mathrm{CDCl}_{3}, 100 \mathrm{MHz}\right): \delta$ 172.2, 154.75, 149.26, 147.95, 135.83, 130.10, 127.12, 112.82, 112.56, 56.38, 56.30, 48.06, 33.36, 32.23, -0.39; LRMS (m/z, relative intensity) : $319\left(\mathrm{M}^{+}, 5\right), 304(100), 288(20), 164(6)$; HRMS calcd. For $\mathrm{C}_{17} \mathrm{H}_{25} \mathrm{NO}_{3} \mathrm{Si}: \mathrm{m} / \mathrm{z} 319.1604$, found $\mathrm{m} / \mathrm{z} 319.1604$

1-Benzylidene-7,8-dimethoxy-1,3,4,5-tetrahydrobenzo[d]azepin-2-one (6i). Synthesized from amide 3i $155 \mathrm{mg}$. Eluent DCM / $\mathrm{Et}_{2} \mathrm{O}(4 / 1)\left(R_{f} 0.24\right)$. Yield: $48 \mathrm{mg}(39 \%)$. ${ }^{1} \mathrm{H} \mathrm{NMR}\left(\mathrm{CDCl}_{3}, 300 \mathrm{MHz}\right): \delta 7.58(\mathrm{~d}, 2 \mathrm{H}, J=7.5 \mathrm{~Hz}), 7.26-$ $7.37(\mathrm{~m}, 3 \mathrm{H}), 6.92(\mathrm{~s}, 1 \mathrm{H}), 6.69(\mathrm{~s}, 1 \mathrm{H}), 6.59(\mathrm{~s}, 1 \mathrm{H}), 6.47(\mathrm{~m}, 1 \mathrm{H}), 3.92(\mathrm{~s}, 3 \mathrm{H}), 3.87(\mathrm{~s}, 3 \mathrm{H}), 3.67(\mathrm{~m}, 2 \mathrm{H}), 3.05(\mathrm{~m}$, $2 \mathrm{H}) ;{ }^{13} \mathrm{C}$ NMR $\left(\mathrm{CDCl}_{3}, 75 \mathrm{MHz}\right): \delta 174.30,149.42,148.13,137.78,135.80,132.41,128.94,128.49,128.43,128.00$, 113.24, 112.69, 56.46, 56.38, 39.81, 34.69; HRMS calcd. For $\mathrm{C}_{19} \mathrm{H}_{19} \mathrm{NO}_{3}: \mathrm{m} / \mathrm{z} 309.1365$, found $\mathrm{m} / \mathrm{z} 309.1362$

1-Ethylidene-7,8-dimethoxy-1,3,4,5-tetrahydro-benzo[d]azepin-2-one (6j). Synthesized from amide 3j $130 \mathrm{mg}$. Eluent DCM / $\mathrm{Et}_{2} \mathrm{O}(20 / 1)\left(R_{f} 0.29\right)$. Yield: $52 \mathrm{mg}(53 \%)$. ${ }^{1} \mathrm{H}$ NMR $\left(\mathrm{CDCl}_{3}, 300 \mathrm{MHz}\right): \delta 6.95(\mathrm{bs}, 1 \mathrm{H}), 6.73(\mathrm{~s}, 1 \mathrm{H})$, $6.54(\mathrm{~s}, 1 \mathrm{H}), 5.97(\mathrm{q}, 1 \mathrm{H}, J=6.9 \mathrm{~Hz}), 3.86(\mathrm{~s}, 3 \mathrm{H}), 3.84(\mathrm{~s}, 3 \mathrm{H}), 3.54(\mathrm{~m}, 2 \mathrm{H}), 2.99(\mathrm{~m}, 2 \mathrm{H}), 1.96(\mathrm{~d}, 3 \mathrm{H}, J=6.9 \mathrm{~Hz})$; ${ }^{13} \mathrm{C}$ NMR $\left(\mathrm{CDCl}_{3}, 75 \mathrm{MHz}\right): \delta 174.03,148.96,147.90,138.78,132.42,131.15,128.98,128.82,127.76,113.01$, 56.32, 39.96, 34.79, 15.93; HRMS calcd. For $\mathrm{C}_{14} \mathrm{H}_{17} \mathrm{NO}_{3}: \mathrm{m} / \mathrm{z} 247.1208$, found $\mathrm{m} / \mathrm{z} 247.1213$

1-Hexylidene-3-methyl-1,3,4,5-tetrahydrobenzo[d]azepin-2-one (6k). Synthesized from amide 3k $135 \mathrm{mg}$. Eluent $\mathrm{DCM} / \mathrm{Et}_{2} \mathrm{O}(4 / 1)\left(R_{f} 0.50\right)$. Yield: $83 \mathrm{mg}(81 \%) .{ }^{1} \mathrm{H}$ NMR $\left(\mathrm{CDCl}_{3}, 400 \mathrm{MHz}\right): \delta$ 7.23-7.27 $(\mathrm{m}, 1 \mathrm{H}), 7.15-7.20(\mathrm{~m}, 2 \mathrm{H})$, 7.05-7.09 (m, 1H), $5.86(\mathrm{t}, 1 \mathrm{H}, J=7.5 \mathrm{~Hz}), 3.70(\mathrm{bs}, 2 \mathrm{H}), 3.05-3.10(\mathrm{~m}, 5 \mathrm{H}), 2.32(\mathrm{q}, 2 \mathrm{H}, J=7.5 \mathrm{~Hz}), 1.45-1.53(\mathrm{~m}$, $2 \mathrm{H}), 1.29-1.37(\mathrm{~m}, 4 \mathrm{H}), 0.89(\mathrm{t}, 3 \mathrm{H}, \mathrm{J}=7.1 \mathrm{~Hz}) ;{ }^{13} \mathrm{C}$ NMR $\left(\mathrm{CDCl}_{3}, 75 \mathrm{MHz}\right): \delta 171.13,139.24,136.77,135.86$, 135.24, 130.31, 130.23, 127.94, 127.00, 47.99, 33.23, 32.26, 31.89, 30.20, 29.39, 22.88, 14.44; HRMS calcd. For $\mathrm{C}_{17} \mathrm{H}_{23} \mathrm{NO}: \mathrm{m} / \mathrm{z} 257.1780$, found $\mathrm{m} / \mathrm{z} 257.1785$

1-(4-Methoxybenzylidene)-3-methyl-1,3,4,5-tetrahydrobenzo[d]azepin-2-one (6I). Synthesized from amide 3 I 149 mg. Eluent DCM / $\mathrm{Et}_{2} \mathrm{O}(4 / 1)\left(R_{f} 0.41\right)$. Yield: $94 \mathrm{mg}(80 \%)$. ${ }^{1} \mathrm{H}$ NMR $\left(\mathrm{CDCl}_{3}, 400 \mathrm{MHz}\right): \delta 7.48(\mathrm{~d}, 2 \mathrm{H}, \mathrm{J}=8.7 \mathrm{~Hz})$, 7.40-7.43 (m, 1H), 7.21-7.24 (m, 2H), 7.09-7.13 (m, 1H), $6.87(\mathrm{~d}, 2 \mathrm{H}, J=8.7 \mathrm{~Hz}), 6.59(\mathrm{~s}, 1 \mathrm{H}), 3.81(\mathrm{~s}, 3 \mathrm{H}), 3.10-3.17$ $(\mathrm{m}, 5 \mathrm{H}) ;{ }^{13} \mathrm{C}$ NMR $\left(\mathrm{CDCl}_{3}, 75 \mathrm{MHz}\right): \delta 171.56,159.93,136.71,136.56,135.65,132.01,130.65,130.55,129.91$, 128.67, 128.27, 127.21, 114.44, 55.68, 47.92, 33.02, 31.87; HRMS calcd. For $\mathrm{C}_{19} \mathrm{H}_{19} \mathrm{NO}_{2}: \mathrm{m} / \mathrm{z} 293.1416$, found $\mathrm{m} / \mathrm{z}$ 293.1419

1-Ethylidene-3-methyl-1,3,4,5-tetrahydrobenzo[d]azepin-2-one (6m). Synthesized from amide $3 \mathrm{~m} 112 \mathrm{mg}$. Eluent $\mathrm{DCM} / \mathrm{Et}_{2} \mathrm{O}(4 / 1)\left(R_{f} 0.32\right)$. Yield: $49 \mathrm{mg}(61 \%) .{ }^{1} \mathrm{H}$ NMR $\left(\mathrm{CDCl}_{3}, 300 \mathrm{MHz}\right): \delta 7.06-7.31(\mathrm{~m}, 4 \mathrm{H}), 5.97(\mathrm{q}, 1 \mathrm{H}, J=$ $6.9 \mathrm{~Hz}), 3.72(\mathrm{bs}, 2 \mathrm{H}), 3.02-3.18(\mathrm{~m}, 5 \mathrm{H}), 1.95(\mathrm{~d}, 3 \mathrm{H}, J=6.9 \mathrm{~Hz}) ;{ }^{13} \mathrm{C} \mathrm{NMR}\left(\mathrm{CDCl}_{3}, 75 \mathrm{MHz}\right): \delta 171.04,140.15$, 135.76, 135.25, 130.82, 130.34, 130.12, 127.97, 126.99, 47.98, 33.23, 32.23, 16.08; HRMS calcd. For $\mathrm{C}_{13} \mathrm{H}_{15} \mathrm{NO}: \mathrm{m} / \mathrm{z}$ 201.1154, found $\mathrm{m} / \mathrm{z} 201.1145$ 
3-Isopropyl-1-(4-methoxybenzylidene)-1,3,4,5-tetrahydrobenzo[d]azepin-2-one (6n). Synthesized from amide $3 \mathrm{n}$ $160 \mathrm{mg}$. Eluent DCM / Et $2 \mathrm{O}(4 / 1)\left(R_{f} 0.48\right)$. Yield: $112 \mathrm{mg}(87 \%)$. ${ }^{1} \mathrm{H}$ NMR $\left(\mathrm{CDCl}_{3}, 300 \mathrm{MHz}\right): \delta 7.39-7.50(\mathrm{~m}, 3 \mathrm{H})$, 7.21-7.29 (m, 2H), 7.07-7.14 (s, 1H), $6.87(\mathrm{~d}, 2 \mathrm{H}, J=8.6 \mathrm{~Hz}), 6.56(\mathrm{~s}, 1 \mathrm{H}), 4.96-5.05(\mathrm{~m}, 1 \mathrm{H}), 3.72-3.92(\mathrm{~m}, 5 \mathrm{H}), 3.13$ (bs, 2H), 1.27 (d, $6 \mathrm{H}, J=6.5 \mathrm{~Hz}) ;{ }^{13} \mathrm{C}$ NMR $\left(\mathrm{CDCl}_{3}, 75 \mathrm{MHz}\right): \delta 171.13,159.86,137.41,136.92,135.52,132.05$, 130.46, 129.76, 128.76, 128.18, 127.11, 114.36, 55.68, 44.03, 39.64, 34.90, 20.99; HRMS calcd. For $\mathrm{C}_{21} \mathrm{H}_{23} \mathrm{NO}_{2}: \mathrm{m} / \mathrm{z}$ 321.1729 , found $\mathrm{m} / \mathrm{z} 321.1736$

3-Isopropyl-1-(3,4,5-trimethoxybenzylidene)-1,3,4,5-tetrahydrobenzo[d]azepin-2-one (60). Synthesized from amide $30184 \mathrm{mg}$. Eluent DCM / Et $2 \mathrm{O}(4 / 1)\left(R_{f} 0.44\right)$. Yield: $137 \mathrm{mg}(90 \%)$. ${ }^{1} \mathbf{H} \mathbf{N M R}\left(\mathrm{CDCl}_{3}, 300 \mathrm{MHz}\right): \delta 7.39-7.45$ $(\mathrm{m}, 1 \mathrm{H}), 7.20-7.32(\mathrm{~m}, 2 \mathrm{H}), 7.09-7.17(\mathrm{~m}, 1 \mathrm{H}), 6.87(\mathrm{~s}, 2 \mathrm{H}), 6.53(\mathrm{~s}, 1 \mathrm{H}), 4.92-5.03(\mathrm{~m}, 1 \mathrm{H}), 3.87(\mathrm{~s}, 9 \mathrm{H}), 3.14(\mathrm{bs}$, $2 \mathrm{H}), 1.26(\mathrm{~d}, 6 \mathrm{H}, J=6.7 \mathrm{~Hz}) ;{ }^{13} \mathrm{C}$ NMR $\left(\mathrm{CDCl}_{3}, 75 \mathrm{MHz}\right): \delta 170.74,153.43,138.81,138.53,136.83,135.52,132.36$, $131.51,130.43,129.86,128.38,127.11,106.49,61.25,56.48,44.22,39.74,34.76,21.09$; HRMS calcd. For $\mathrm{C}_{23} \mathrm{H}_{27} \mathrm{NO}_{4}: \mathrm{m} / \mathrm{z} 381.1940$, found $\mathrm{m} / \mathrm{z} 381.1942$

1-Ethylidene-8,9-dimethoxy-3-(4-methoxybenzyl)-3,4,5,6-tetrahydro-1H-benzo[d]azocin-2-one

(11a). Synthesized from amide 10a $184 \mathrm{mg}$. Eluent DCM / $\mathrm{Et}_{2} \mathrm{O}(4 / 1)\left(R_{f} 0.30\right)$. Yield: $84 \mathrm{mg}(55 \%) .{ }^{1} \mathrm{H} \mathbf{N M R}\left(\mathrm{CDCl}_{3}, 400\right.$ $\mathrm{MHz}): \delta 7.26(\mathrm{~d}, 2 \mathrm{H}, J=8.6 \mathrm{~Hz}), 7.01(\mathrm{~s}, 1 \mathrm{H}), 6.87(\mathrm{~d}, 2 \mathrm{H}, J=8.6 \mathrm{~Hz}), 6.56(\mathrm{~s}, 1 \mathrm{H}), 5.92(\mathrm{q}, 1 \mathrm{H}, J=6.9 \mathrm{~Hz}), 4.59(\mathrm{~s}$, $2 \mathrm{H}), 3.67(\mathrm{~s}, 3 \mathrm{H}), 3.85(\mathrm{~s}, 3 \mathrm{H}), 3.80(\mathrm{~s}, 3 \mathrm{H}), 3.43(\mathrm{t}, 2 \mathrm{H}, J=6.2 \mathrm{~Hz}), 2.62-2.66(\mathrm{~m}, 2 \mathrm{H}), 1.80-1.89(\mathrm{~m}, 5 \mathrm{H}) ;{ }^{13} \mathrm{C} \mathrm{NMR}$ $\left(\mathrm{CDCl}_{3}, 75 \mathrm{MHz}\right): \delta 172.71,159.44,148.74,147.99,140.00,130.95,130.25,129.95,129.46,124.71,114.65,114.23$, 114.46, 56.31, 55.65, 46.73, 45.20, 31.82, 29.21, 15.42; HRMS calcd. For $\mathrm{C}_{23} \mathrm{H}_{27} \mathrm{NO}_{4}: \mathrm{m} / \mathrm{z} 381.1940$, found $\mathrm{m} / \mathrm{z}$ 381.1952

1-Benzylidene-8,9-dimethoxy-3-(4-methoxybenzyl)-3,4,5,6-tetrahydro-1H-benzo[d]azocin-2-one

(11b). Synthesized from amide 10b $209 \mathrm{mg}$. Eluent DCM / $\mathrm{Et}_{2} \mathrm{O}(20 / 1)\left(R_{f} 0.24\right)$. Yield: $62 \mathrm{mg}(35 \%) .{ }^{1} \mathrm{H}$ NMR $\left(\mathrm{CDCl}_{3}, 400\right.$ $\mathrm{MHz})$ : $\delta$ 7.49-7.52 (m, 2H), 7.30-7.34 (m, 3H), 7.13 (s, 1H), $6.99(\mathrm{~d}, 2 \mathrm{H}, J=8.5 \mathrm{~Hz}), 6.77(\mathrm{~d}, 2 \mathrm{H}, J=8.5 \mathrm{~Hz}), 6.69(\mathrm{~s}$, $1 \mathrm{H}), 6.64(\mathrm{~s}, 1 \mathrm{H}), 4.56(\mathrm{~s}, 2 \mathrm{H}), 3.94(\mathrm{~s}, 3 \mathrm{H}), 3.90(\mathrm{~s}, 3 \mathrm{H}), 3.80(\mathrm{~s}, 3 \mathrm{H}), 3.39(\mathrm{~m}, 2 \mathrm{H}), 2.74-2.78(\mathrm{~m}, 2 \mathrm{H}), 1.85-1.91(\mathrm{~m}$, $2 \mathrm{H}) ;{ }^{13} \mathrm{C}$ NMR $\left(\mathrm{CDCl}_{3}, 100 \mathrm{MHz}\right): \delta 171.97,159.01,148.81,147.84,138.86,135.82,131.65,130.67,129.70,128.59$, 128.52, 128.43, 128.32, 127.85, 114.35, 113.90, 112.36, 56.10, 56.02, 55.25, 46.89, 45.85, 32.43, 28.99; HRMS calcd. For $\mathrm{C}_{28} \mathrm{H}_{29} \mathrm{NO}_{4}$ : $\mathrm{m} / \mathrm{z} 443.2097$, found $\mathrm{m} / \mathrm{z} 443.2105$

\section{Hydrogenation.}

Compounds $6 \mathbf{a}, \mathbf{g}, \mathbf{m}, \mathbf{o}$ were hydrogenated under atmospheric pressure of $\mathrm{H}_{2}$ over (10\%) $\mathrm{Pd} / \mathrm{C}(5 \mathrm{~mol} \%)$ using THF / $\mathrm{MeOH}(1 / 1)$ mixture as the solvent to give the compounds 9a-d respectively. After completion of the reaction indicated by MS analysis the catalyst was filtered off, the filtrate was concentrated and applied to a silica gel column. Elution with DCM / $\mathrm{Et}_{2} \mathrm{O}(4 / 1)$ mixture afforded compounds 9a-d.

1-Benzyl-7,8-dimethoxy-3-(4-methoxybenzyl)-1,3,4,5-tetrahydrobenzo[d]azepin-2-one (9a). Eluent DCM / $\mathrm{Et}_{2} \mathrm{O}$ (4 / 1) $\left(R_{f} 0.46\right)$. Yield: $90 \% .{ }^{1} \mathrm{H}$ NMR $\left(\mathrm{CDCl}_{3}, 400 \mathrm{MHz}\right): \delta 7.24-7.28(\mathrm{~m}, 4 \mathrm{H}), 7.17-7.21(\mathrm{~m}, 1 \mathrm{H}), 6.95(\mathrm{~d}, 2 \mathrm{H}, J=8.4$ $\mathrm{Hz}), 6.74(\mathrm{~d}, 2 \mathrm{H}, J=8.4 \mathrm{~Hz}), 6.61(\mathrm{~s}, 1 \mathrm{H}), 6.47(\mathrm{~s}, 1 \mathrm{H}), 4.60(\mathrm{~d}, 1 \mathrm{H}, J=14.6 \mathrm{~Hz}), 4.46(\mathrm{t}, 1 \mathrm{H}, J=7.0 \mathrm{~Hz}), 3.81-3.87$ $(\mathrm{m}, 1 \mathrm{H}), 3.79(\mathrm{~s}, 3 \mathrm{H}), 3.76(\mathrm{~s}, 3 \mathrm{H}), 3.75(\mathrm{~s}, 3 \mathrm{H}), 3.59-3.64\left(\mathrm{dd}, 1 \mathrm{H}, J_{1}=8.3 \mathrm{~Hz}, J_{2}=13.6 \mathrm{~Hz}\right), 3.41-3.48\left(\mathrm{dt}, 1 \mathrm{H}, J_{1}=\right.$ $\left.6.0 \mathrm{~Hz}, J_{2}=15.2 \mathrm{~Hz}\right), 3.19-3.24\left(\mathrm{dd}, 1 \mathrm{H}, J_{1}=6.3 \mathrm{~Hz}, J_{2}=13.6 \mathrm{~Hz}\right), 2.99-3.06\left(\mathrm{dt}, 1 \mathrm{H}, J_{1}=5.6 \mathrm{~Hz}, J_{2}=16.9 \mathrm{~Hz}\right), 2.75-$ $2.84(\mathrm{~m}, 1 \mathrm{H}) ;{ }^{13} \mathrm{C}$ NMR $\left(\mathrm{CDCl}_{3}, 100 \mathrm{MHz}\right): \delta 173.01,159.06,147.83,147.40,140.16,129.91,129.34,128.43$, 128.30, 128.27, 126.28, 114.01, 113.62, 111.17, 56.07, 56.01, 55.34, 50.21, 49.57, 45.69, 36.41, 32.43; HRMS calcd. For $\mathrm{C}_{27} \mathrm{H}_{29} \mathrm{NO}_{4}: \mathrm{m} / \mathrm{z}$ 431.2097, found $\mathrm{m} / \mathrm{z} 431.2095$

1-Benzyl-7,8-dimethoxy-3-methyl-1,3,4,5-tetrahydrobenzo[d]azepin-2-one (9b). Eluent DCM / $\mathrm{Et}_{2} \mathrm{O}(4 / 1)\left(R_{f}\right.$ 0.43). Yield: $96 \%$. ${ }^{1} \mathrm{H}$ NMR $\left(\mathrm{CDCl}_{3}, 400 \mathrm{MHz}\right): \delta 7.24-7.28(\mathrm{~m}, 4 \mathrm{H}), 7.16-7.22(\mathrm{~m}, 1 \mathrm{H}), 6.61(\mathrm{~s}, 1 \mathrm{H}), 6.57(\mathrm{~s}, 1 \mathrm{H}), 4.41$ $(\mathrm{t}, 1 \mathrm{H}, J=7.3 \mathrm{~Hz}), 3.90-3.98(\mathrm{~m}, 1 \mathrm{H}), 3.84(\mathrm{~s}, 3 \mathrm{H}), 3.73(\mathrm{~s}, 3 \mathrm{H}), 3.56-3.62\left(\mathrm{dd}, 1 \mathrm{H}, J_{1}=7.8 \mathrm{~Hz}, J_{2}=13.6 \mathrm{~Hz}\right), 3.48-$ $3.54(\mathrm{~m}, 1 \mathrm{H}), 3.18-3.24(\mathrm{~m}, 2 \mathrm{H}), 3.04-3.12(\mathrm{~m}, 1 \mathrm{H}), 2.97(\mathrm{~s}, 3 \mathrm{H}) ;{ }^{13} \mathbf{C} \mathbf{N M R}\left(\mathrm{CDCl}_{3}, 100 \mathrm{MHz}\right): \delta 173.13,147.92$, 147.49, 140.49, 129.64, 128.73, 128.61, 126.53, 113.46, 111.30, 56.29, 56.23, 49.55, 49.23, 36.61, 35.88, 32.15; HRMS calcd. For $\mathrm{C}_{20} \mathrm{H}_{23} \mathrm{NO}_{3}: \mathrm{m} / \mathrm{z} 325.1678$, found $\mathrm{m} / \mathrm{z} 325.1679$

1-Ethyl-3-methyl-1,3,4,5-tetrahydrobenzo[d]azepin-2-one (9c). Eluent DCM / $\mathrm{Et}_{2} \mathrm{O}(4 / 1)\left(R_{f} 0.32\right)$. Yield: $96 \%$. ${ }^{1} \mathrm{H}$ NMR $\left(\mathrm{CDCl}_{3}, 400 \mathrm{MHz}\right): \delta$ 7.13-7.19 (m, 3H). 7.08-7.12 (m, 2H), 3.87-3.99 (m, 2H), 3.46-3.53 (m, 1H), 3.21-3.28 (m, $1 \mathrm{H}), 3.07-3.14(\mathrm{~m}, 1 \mathrm{H}), 2.96(\mathrm{~s}, 3 \mathrm{H}), 2.20-2.31(\mathrm{~m}, 1 \mathrm{H}), 1.87-1.97(\mathrm{~m}, 1 \mathrm{H}), 1.03(\mathrm{t}, 3 \mathrm{H}, J=7.3 \mathrm{~Hz}) ;{ }^{13} \mathrm{C} \mathrm{NMR}\left(\mathrm{CDCl}_{3}\right.$, $100 \mathrm{MHz}$ ) : $\delta$ 173.08, 137.36, 136.60, 129.85, 127.27, 126.83, 126.81, 50.54, 49.31, 35.81, 32.33, 23.55, 13.02; HRMS calcd. For $\mathrm{C}_{20} \mathrm{H}_{23} \mathrm{NO}_{3}: \mathrm{m} / \mathrm{z} 203.1310$, found $\mathrm{m} / \mathrm{z} 203.1315$

3-Isopropyl-1-(3,4,5-trimethoxybenzyl)-1,3,4,5-tetrahydrobenzo[d]azepin-2-one (9d). Eluent $\mathrm{DCM} / \mathrm{Et}_{2} \mathrm{O}(4 / 1)$ $\left(R_{f}\right.$ 0.44). Yield: $95 \%$. ${ }^{1} \mathrm{H}$ NMR $\left(\mathrm{CDCl}_{3}, 400 \mathrm{MHz}\right): \delta$ 7.11-7.18 $(\mathrm{m}, 3 \mathrm{H}), 7.03-7.08(\mathrm{~m}, 1 \mathrm{H}), 6.48(\mathrm{~s}, 2 \mathrm{H}), 4.74-4.84(\mathrm{~s}$, $1 \mathrm{H}, J=6.7 \mathrm{~Hz}), 4.40-4.45(\mathrm{~m}, 1 \mathrm{H}), 3.77-3.87(\mathrm{~m}, 10 \mathrm{H}), 3.50-3.63(\mathrm{~m}, 2 \mathrm{H}), 3.19-3.27(\mathrm{~m}, 1 \mathrm{H}), 3.03-3.13(\mathrm{~s}, 1 \mathrm{H}) ; 1.08$ $(\mathrm{d}, 3 \mathrm{H}, J=6.7 \mathrm{~Hz}), 1.02(\mathrm{~d}, 3 \mathrm{H}, J=6.7 \mathrm{~Hz}) ;{ }^{13} \mathrm{C} \mathrm{NMR}\left(\mathrm{CDCl}_{3}, 100 \mathrm{MHz}\right): \delta 172.47,152.97,136.54,136.47,136.22$, 
135.70, 130.37, 126.85, 126.80, 126.42, 106.29, 60.77, 56.08, 49.89, 44.60, 38.95, 35.92, 34.08, 20.21; HRMS calcd. For $\mathrm{C}_{13} \mathrm{H}_{17} \mathrm{NO}: \mathrm{m} / \mathrm{z} 203.1310$, found $\mathrm{m} / \mathrm{z} 203.1312$

\section{MW-assisted PMB-deprotection under acidic conditions.}

1-Benzylidene-7,8-dimethoxy-1,3,4,5-tetrahydrobenzo[d]azepin-2-one (6i). 1-Benzylidene-7,8-dimethoxy-3-(4methoxybenzyl)-1,3,4,5-tetrahydrobenzo[d]azepin-2-one (6a) $710 \mathrm{mg}(1.65 \mathrm{mmol})$ was dissolved in the mixture of $\mathrm{DCM}(12 \mathrm{~mL})$, TFA $(4 \mathrm{~mL})$ containing the catalytic amount of TfOH $(100 \mu \mathrm{L})$. The reaction mixture was loaded into the microwave instrument vial and irradiated with stirring at $110^{\circ} \mathrm{C}$ with the maximum power level of $300 \mathrm{~W}$ during $45 \mathrm{~min}$. The volatiles were removed under reduced pressure, the residue was taken up in DCM, washed with $1 \mathrm{M} \mathrm{NaOH}$ and brine. After drying $\left(\mathrm{MgSO}_{4}\right)$ and concentration the crude product was purified by column chromatography on silica gel as described earlier. Yield: $426 \mathrm{mg}(84 \%)$.

1-Ethylidene-7,8-dimethoxy-1,3,4,5-tetrahydro-benzo[d]azepin-2-one (6j). was synthesized similarly as described for (6i) from 1-Ethylidene-7,8-dimethoxy-3-(4-methoxybenzyl)-1,3,4,5-tetrahydrobenzo[d]azepin-2-one (6b) $606 \mathrm{mg}$ (1.65 mmol). Yield: $326 \mathrm{mg}(80 \%)$.

\section{References.}

1. Moreau, A.; Couture, A.; Deniau, E.; Grandclaudon, P. Eur. J. Org. Chem. 2005, 16, 3437

2. Fidalgo, J.; Castedo, L.; Dominiguez, D. Heterocycles 1994, 39, 581

3. Appukkuttan, P.; Orts, A.B.; Chandran, R.P.; Goeman, J. L.; Van der Eycken, J.; Dehaen, W.; Van der Eycken, Erik. Eur. J. Org. Chem. 2004, 15, 3277

4. Cevallos, A.; Rios, R.; Moyano, A.; Pericas, M.A.; Riera, A. Tetrahedron: Asymmetry, 2000, 11, 4407

5. Perkin-Elmer NMR. Quarterly, 1975, 15 

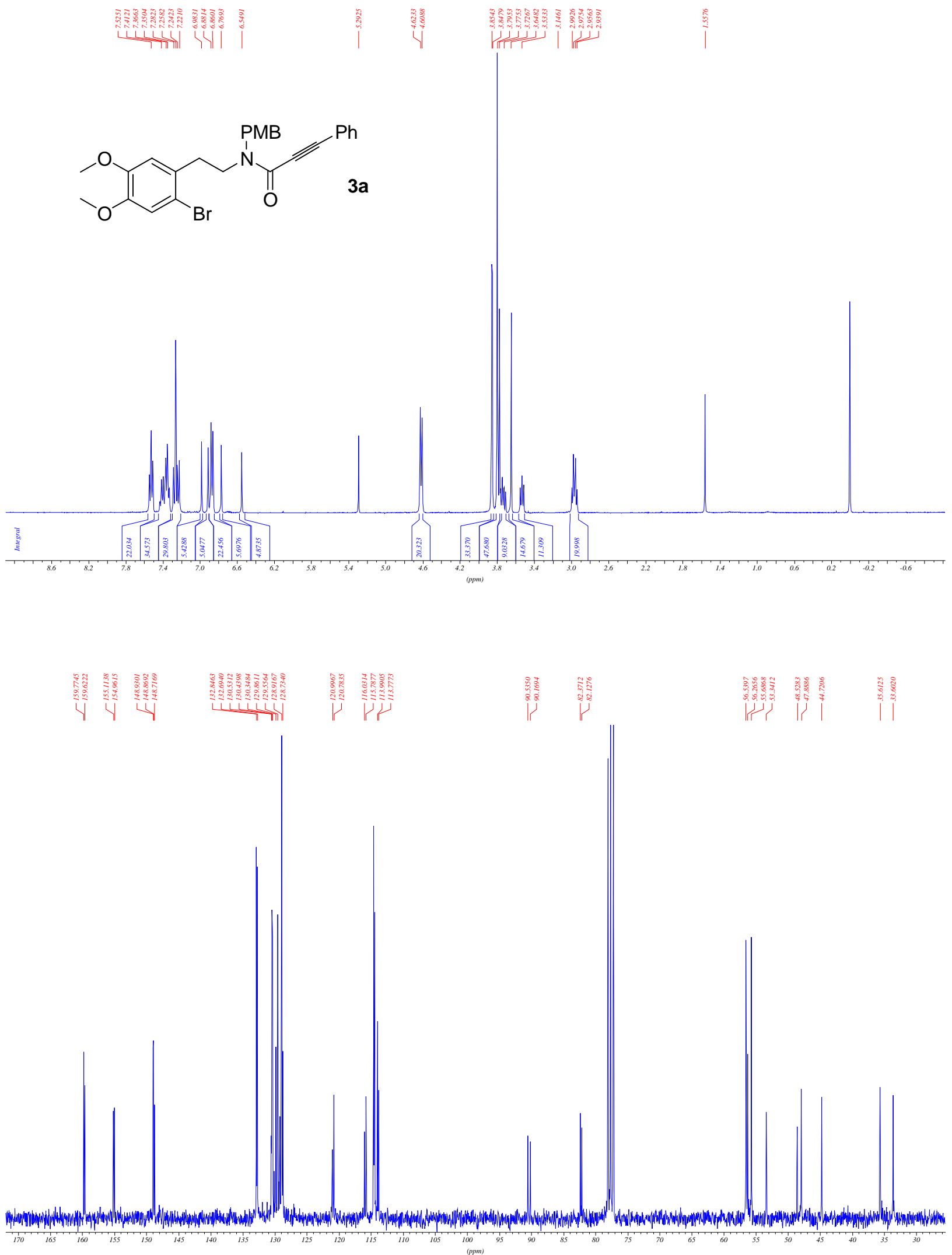

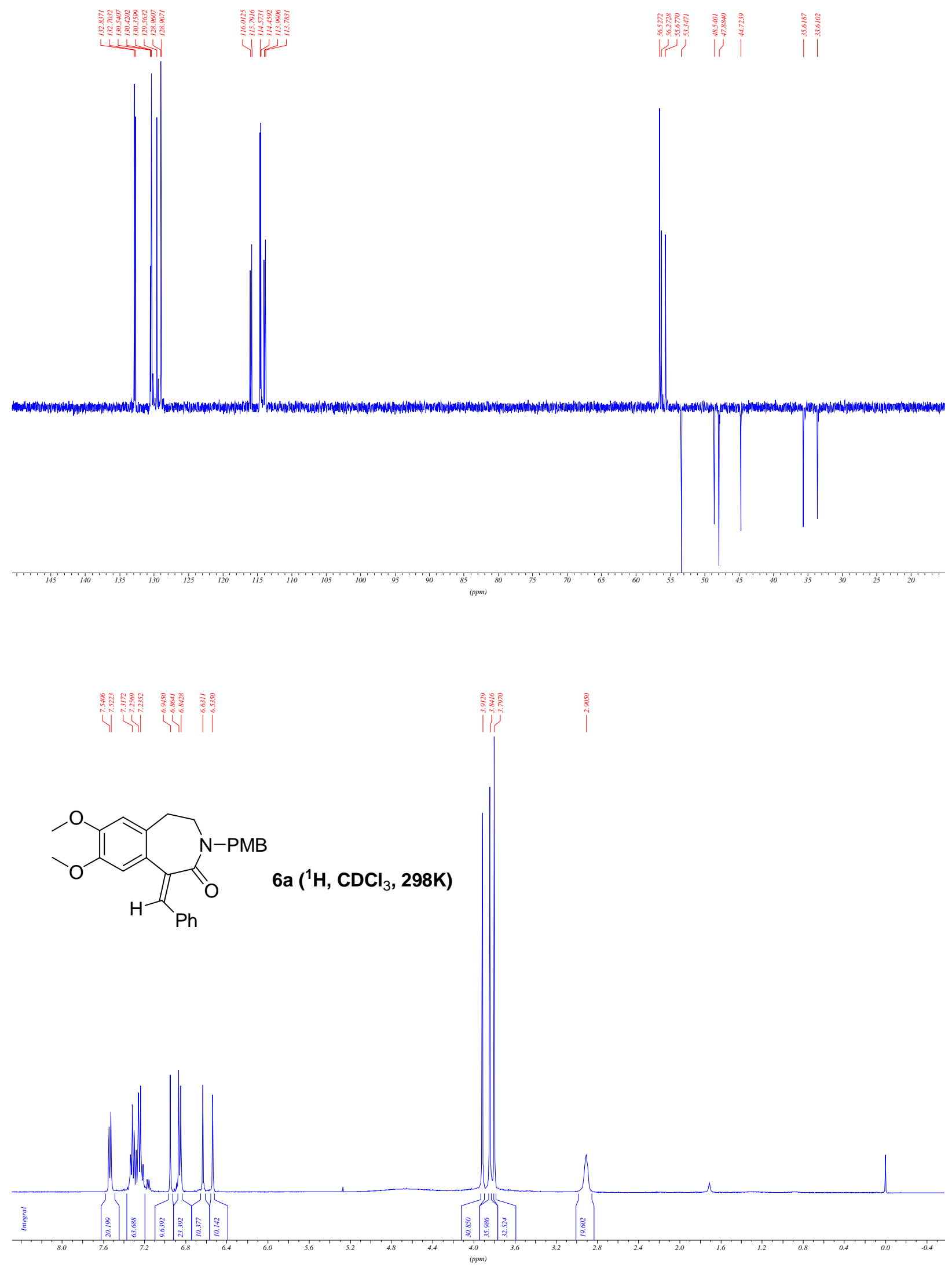

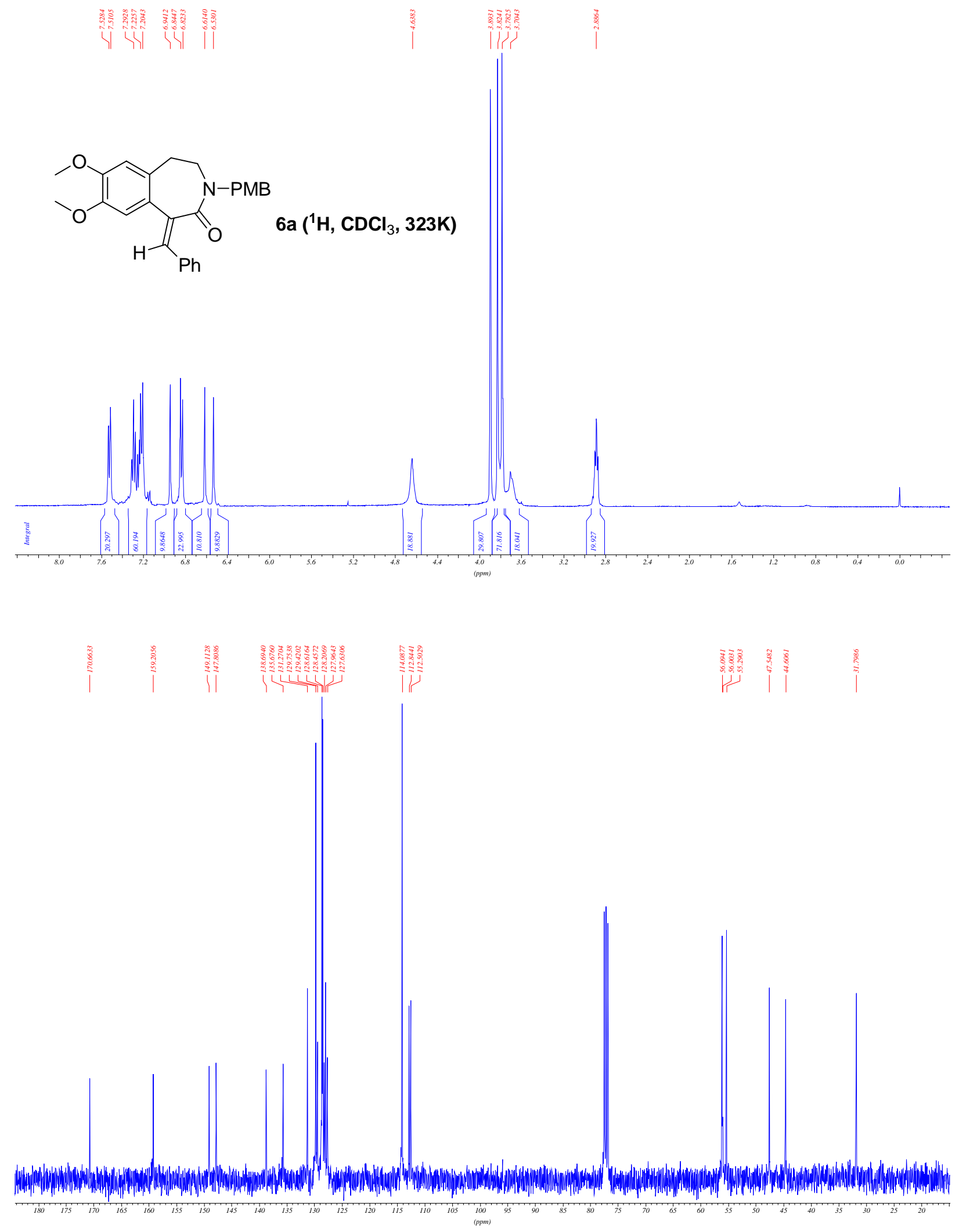

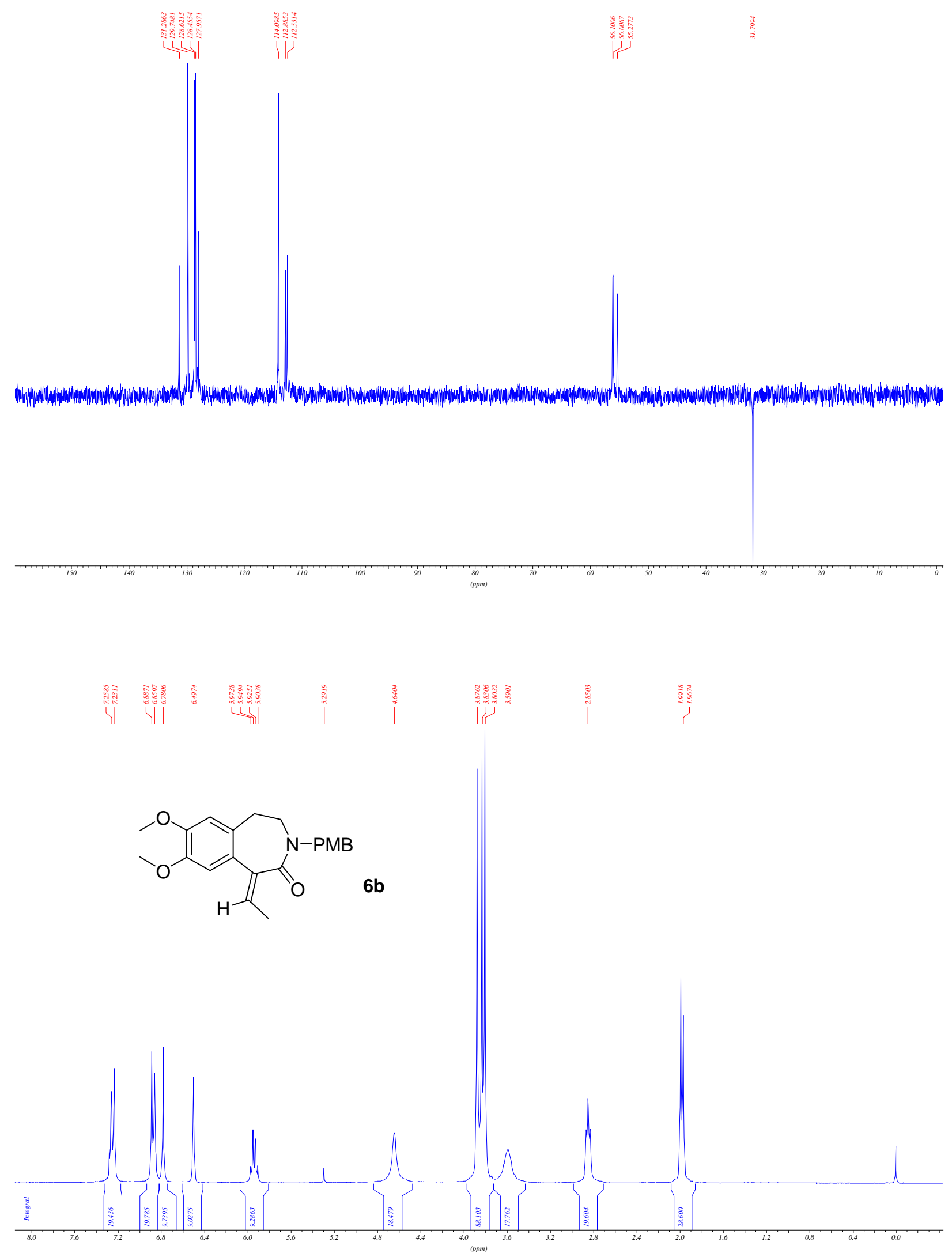

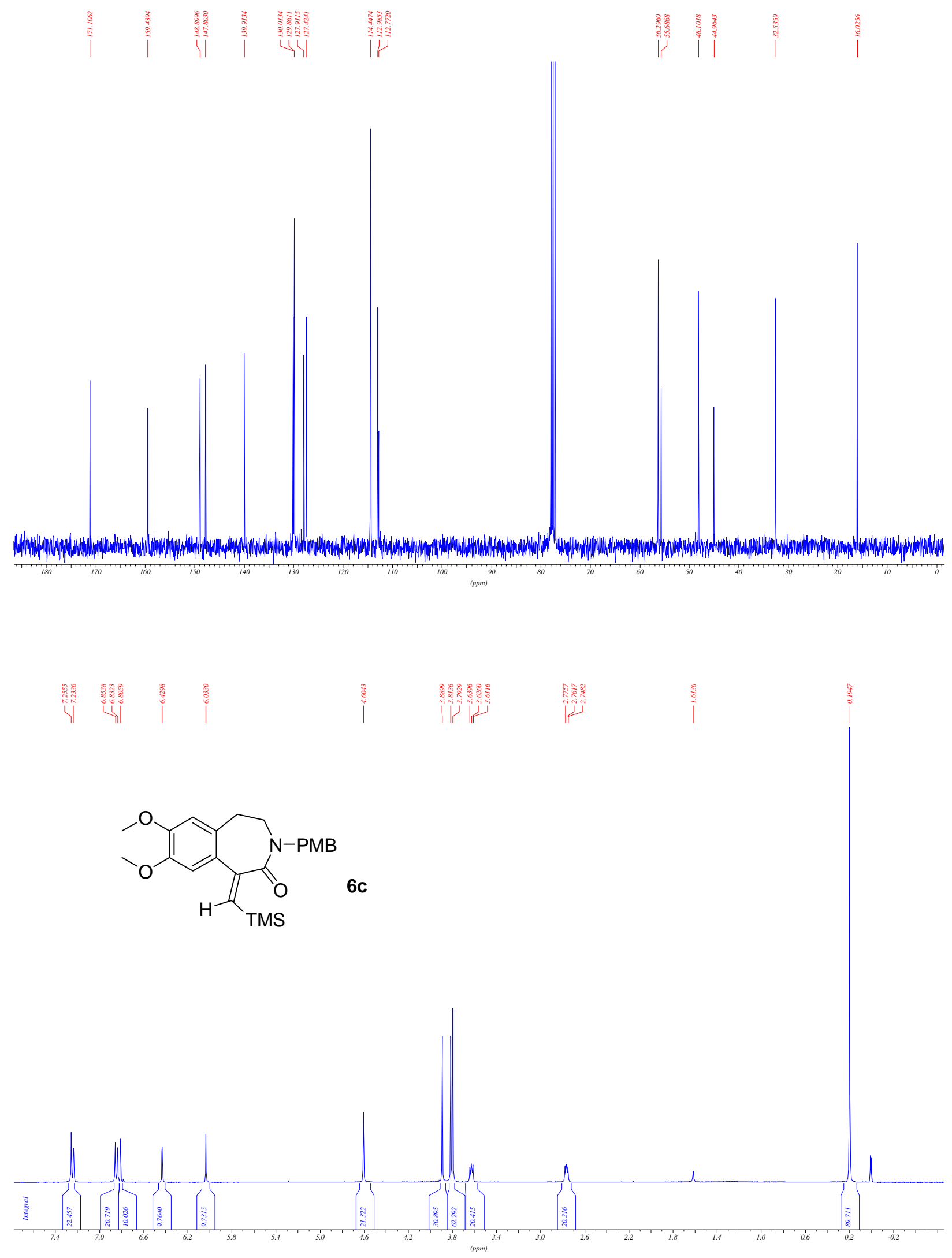


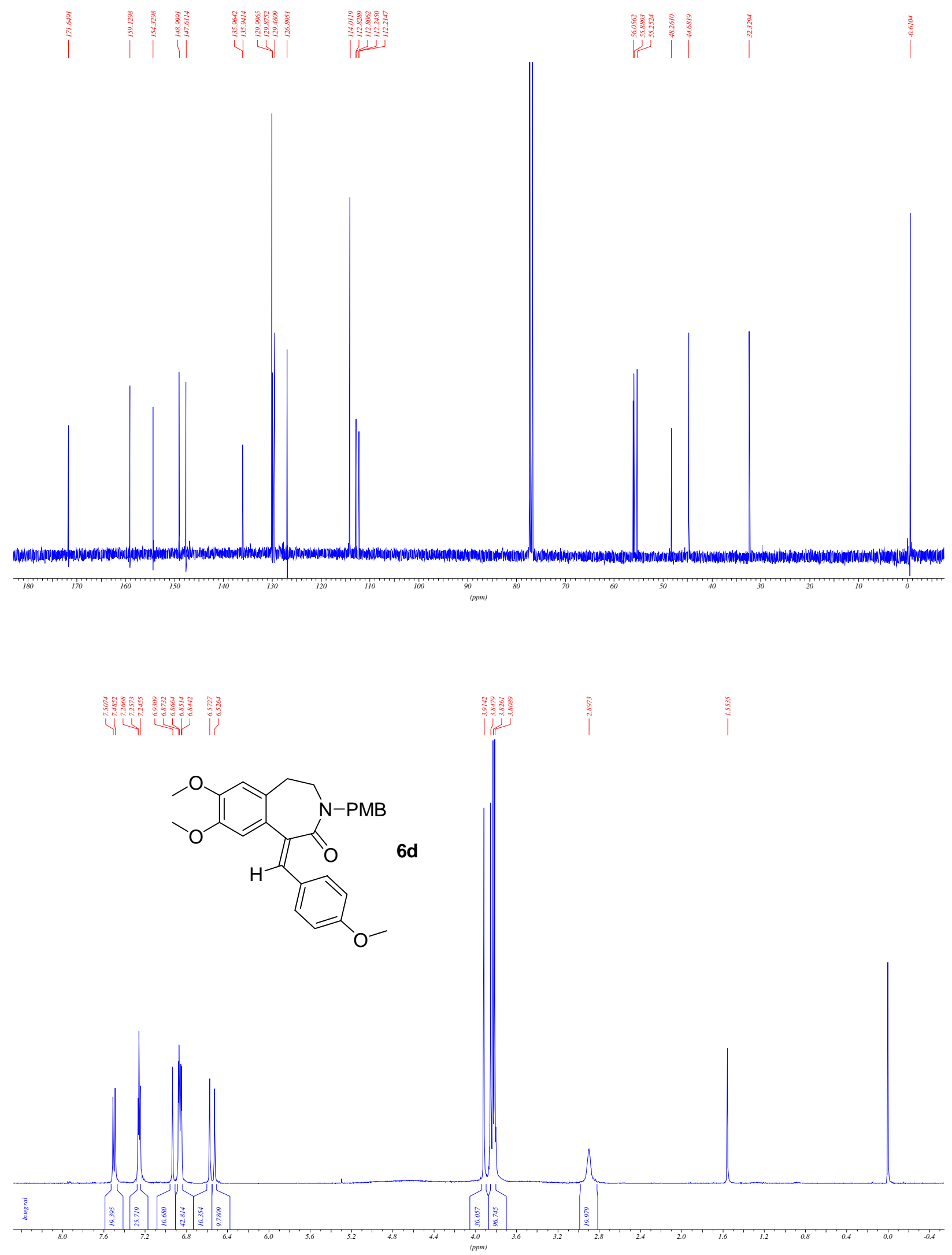



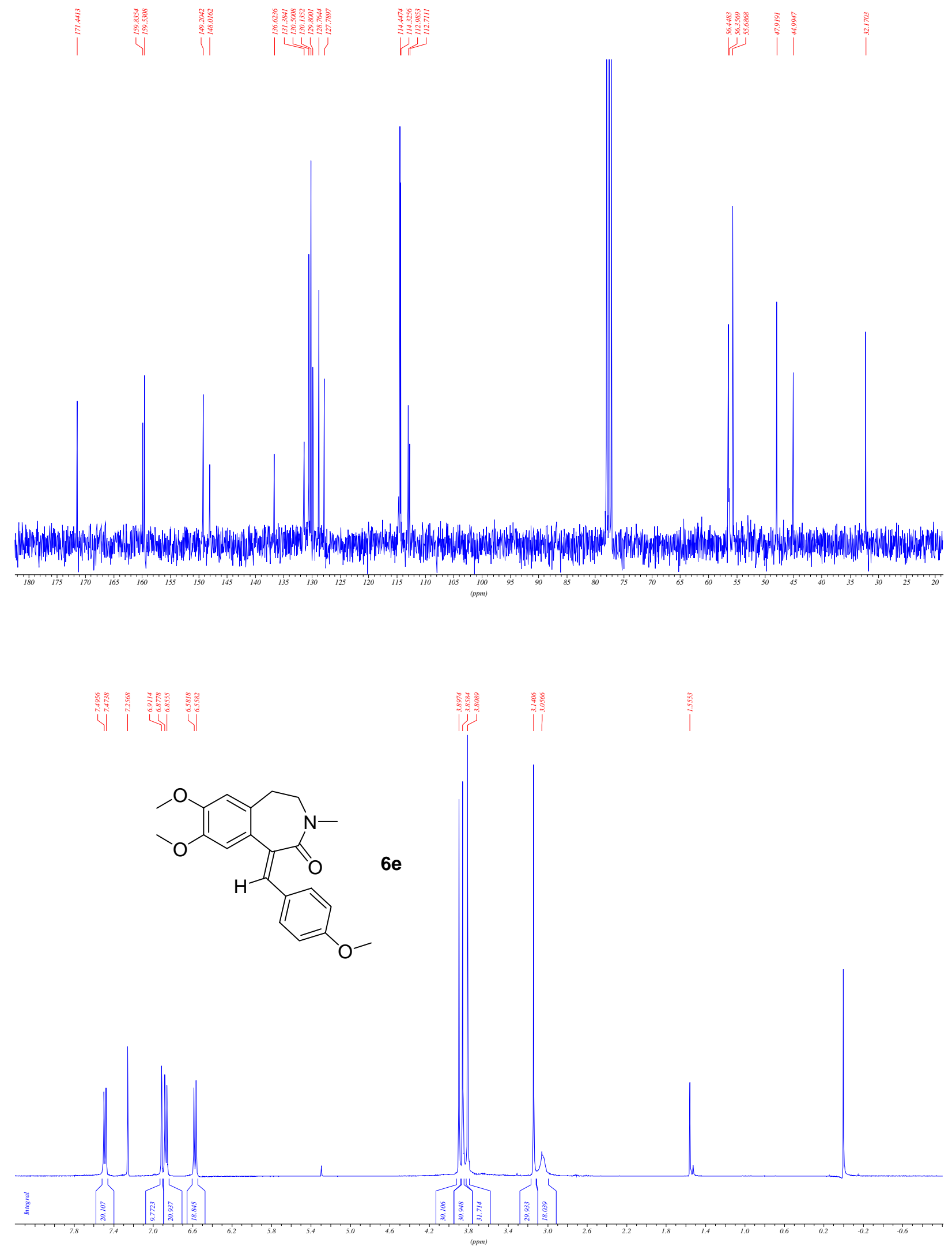


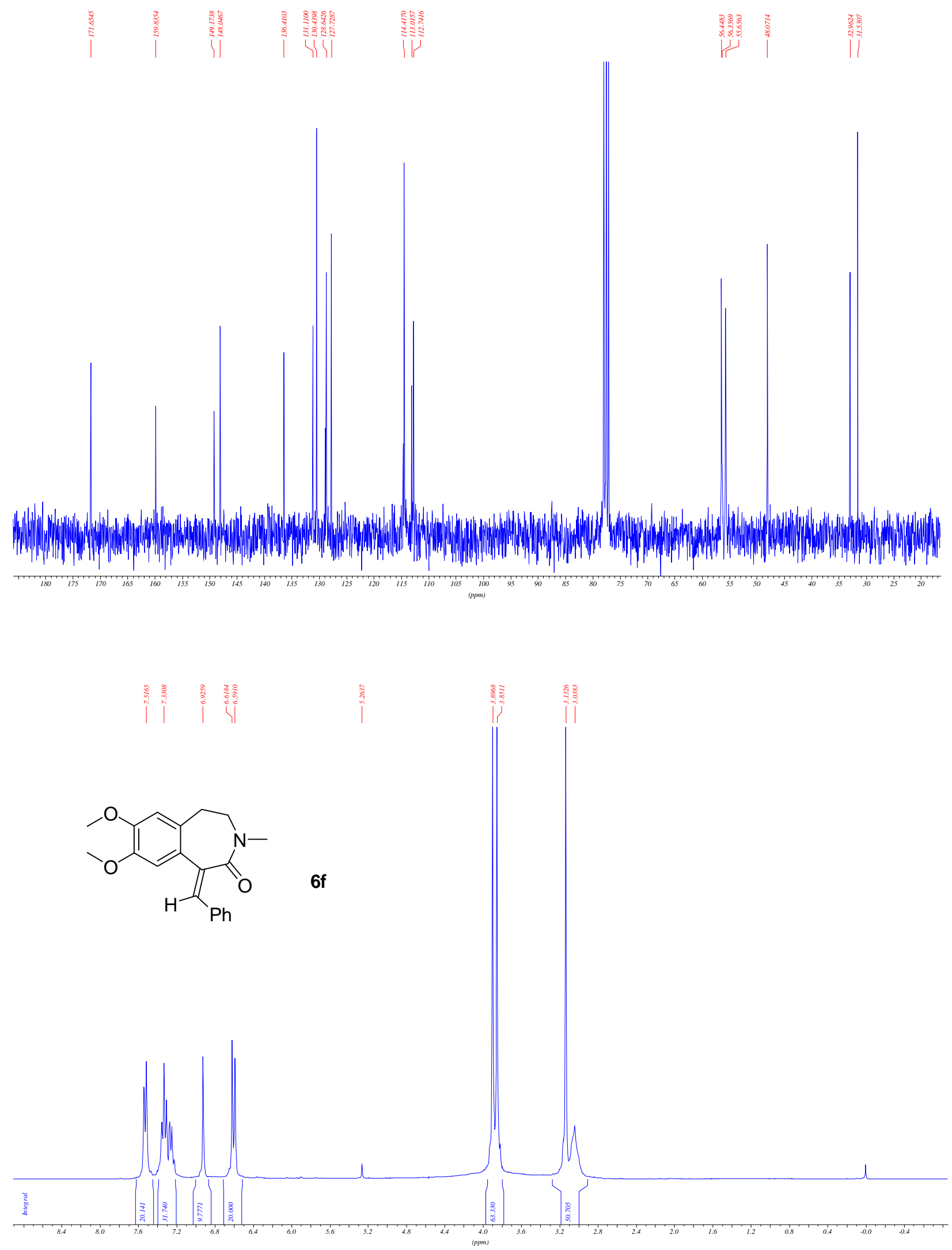




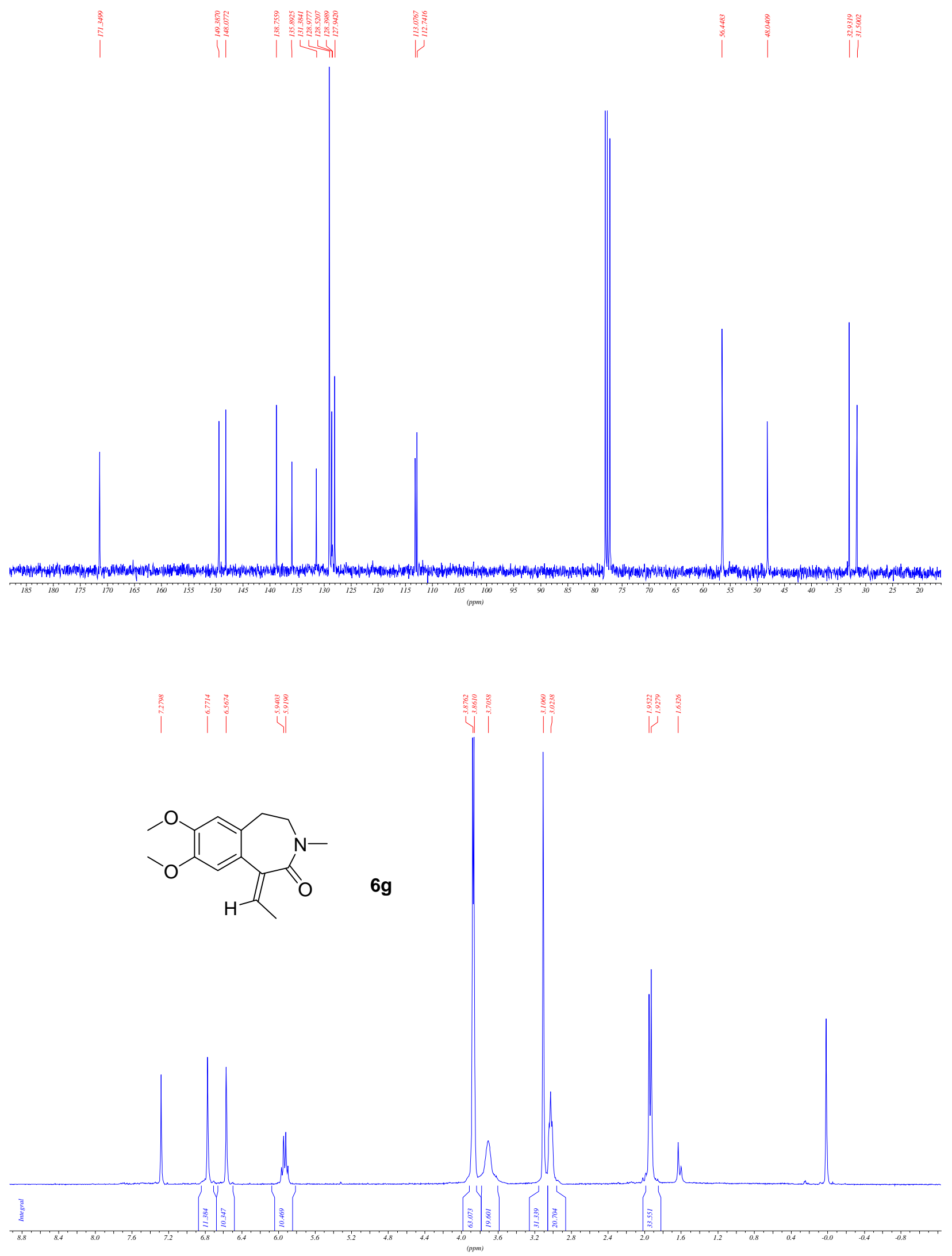




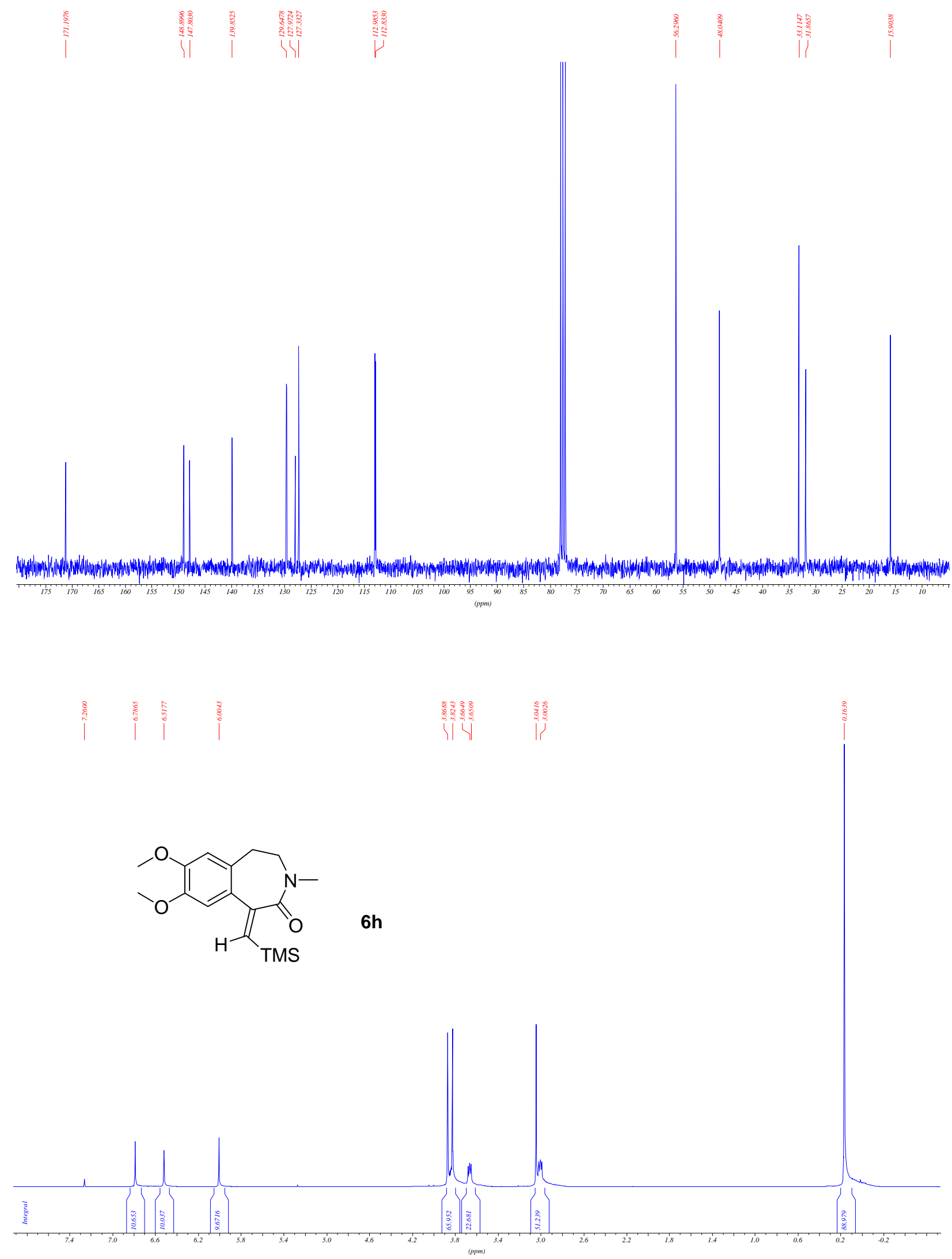



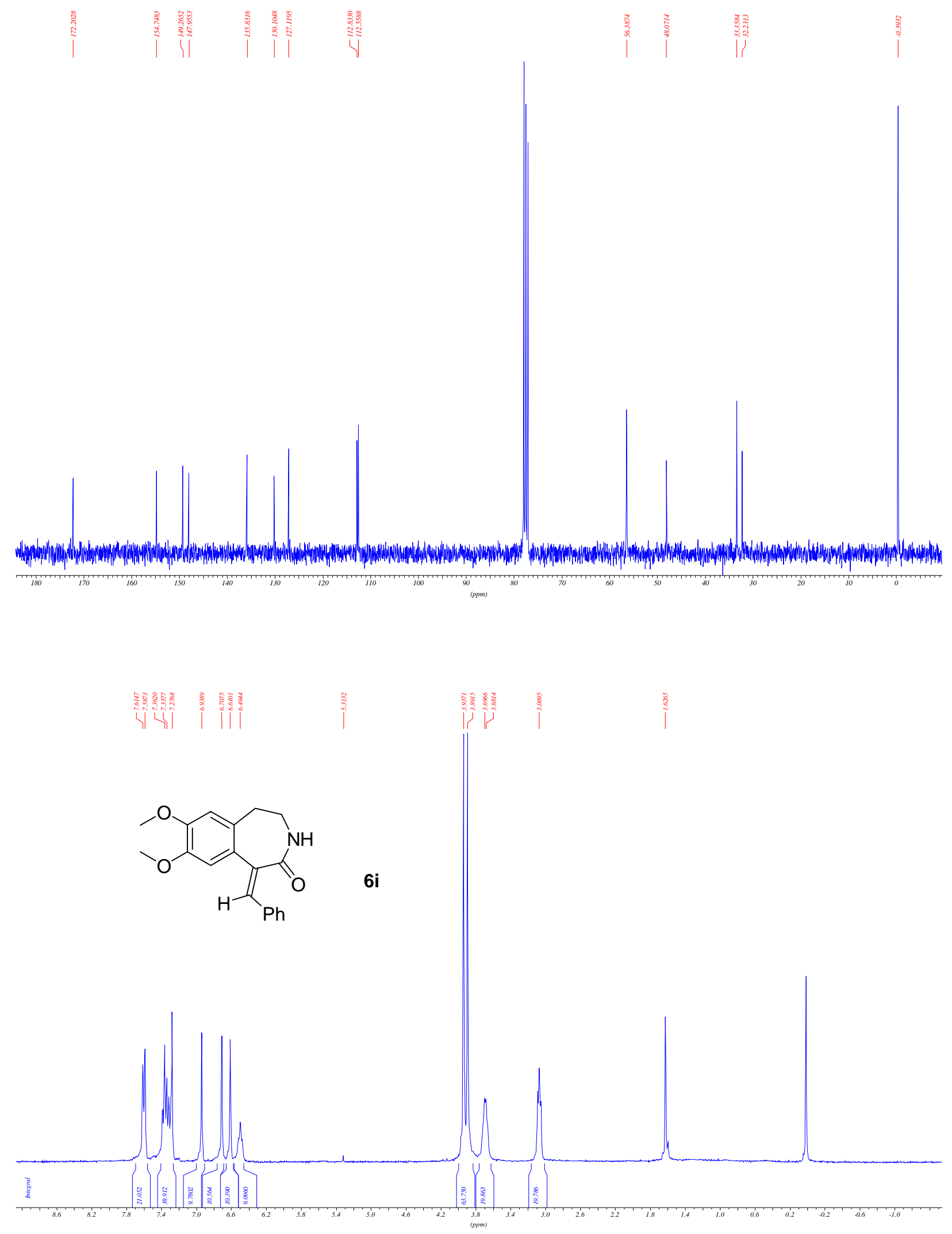


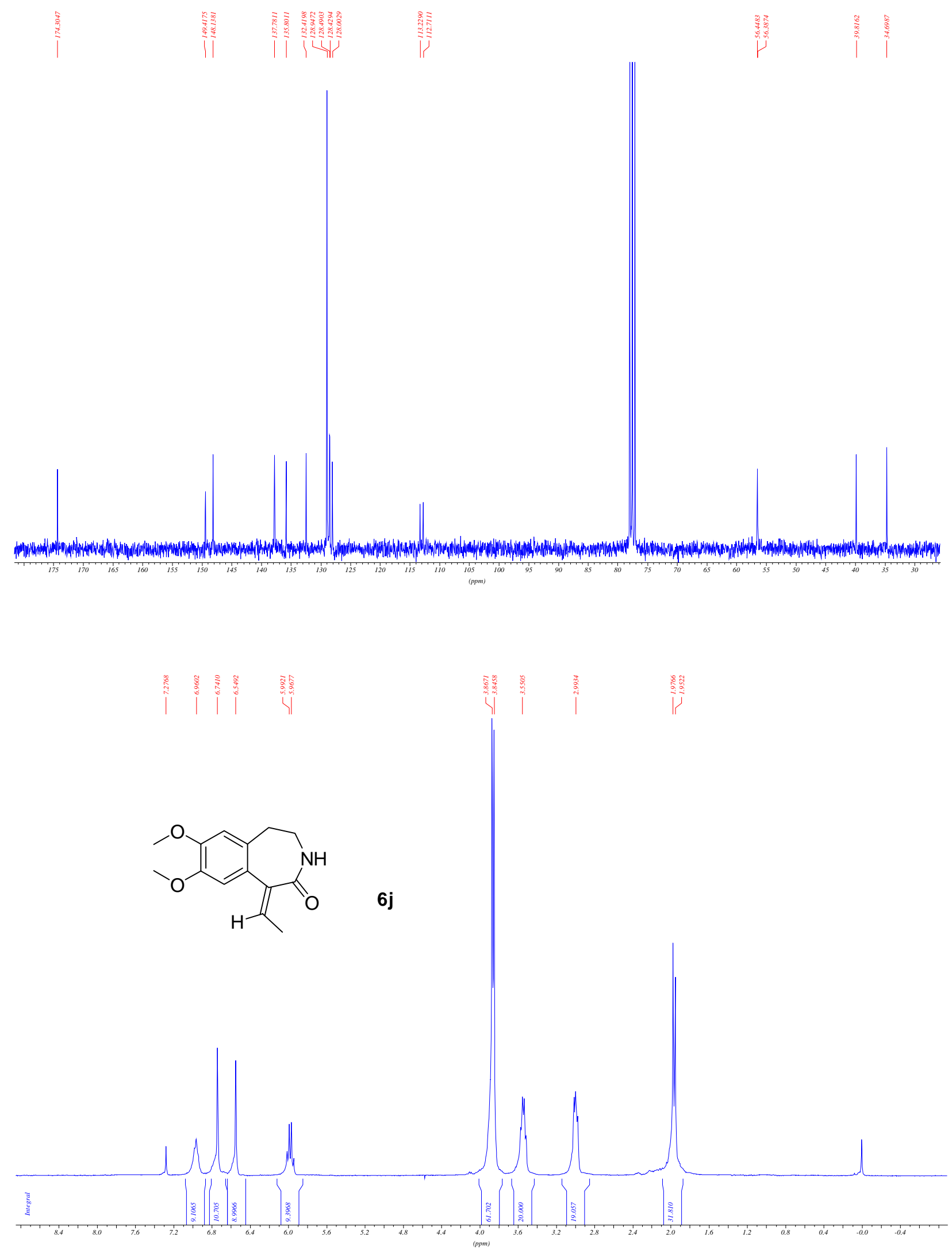



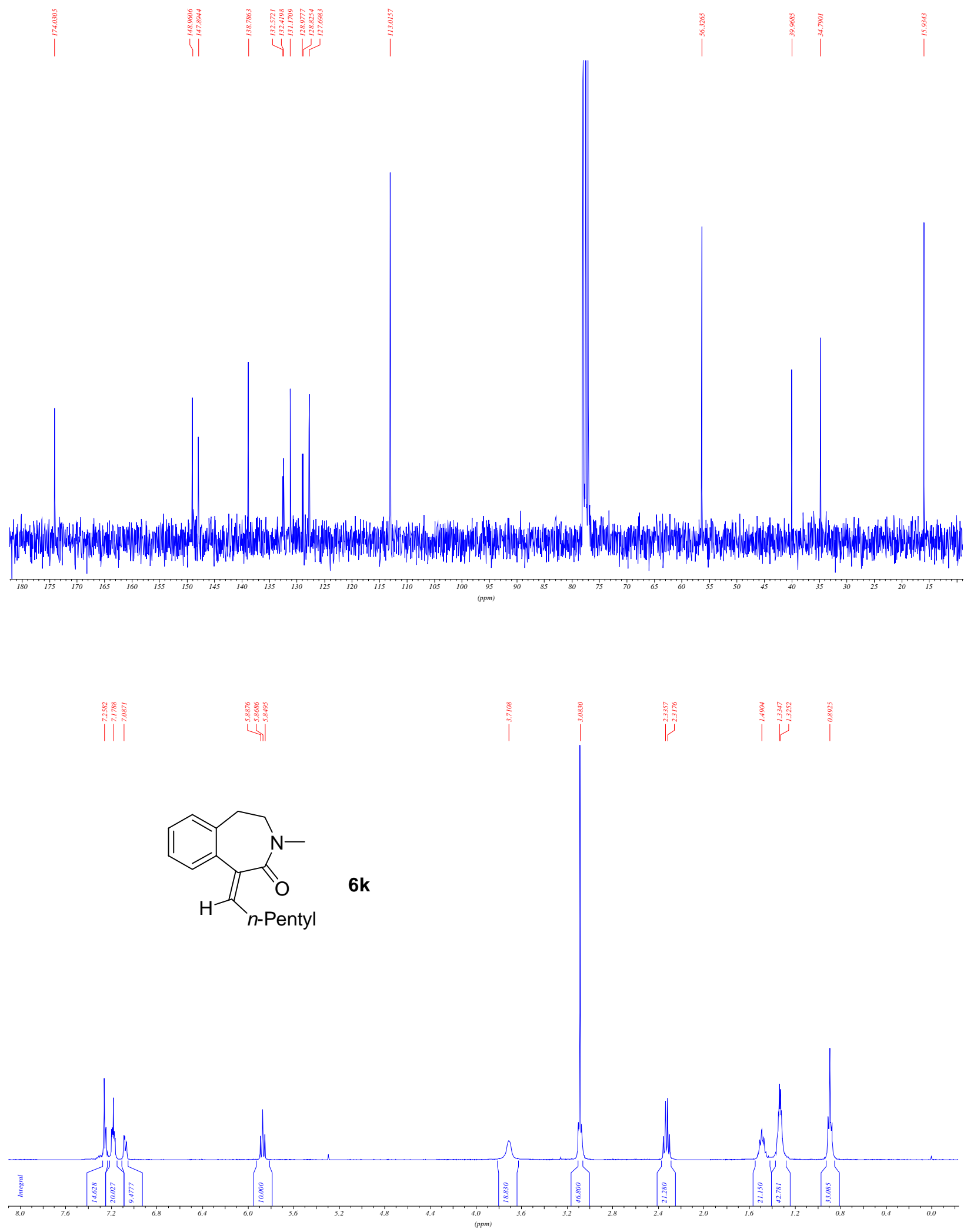

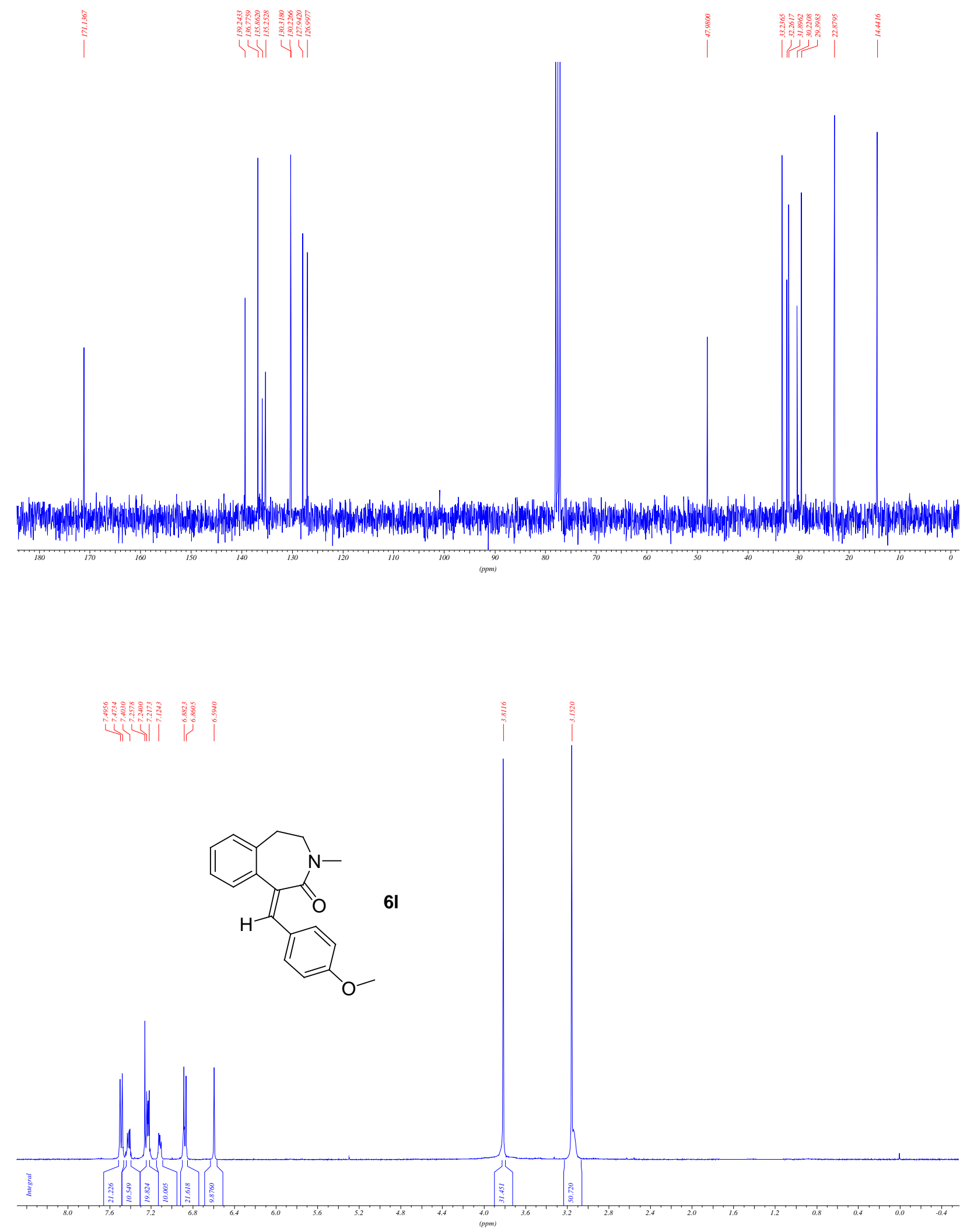


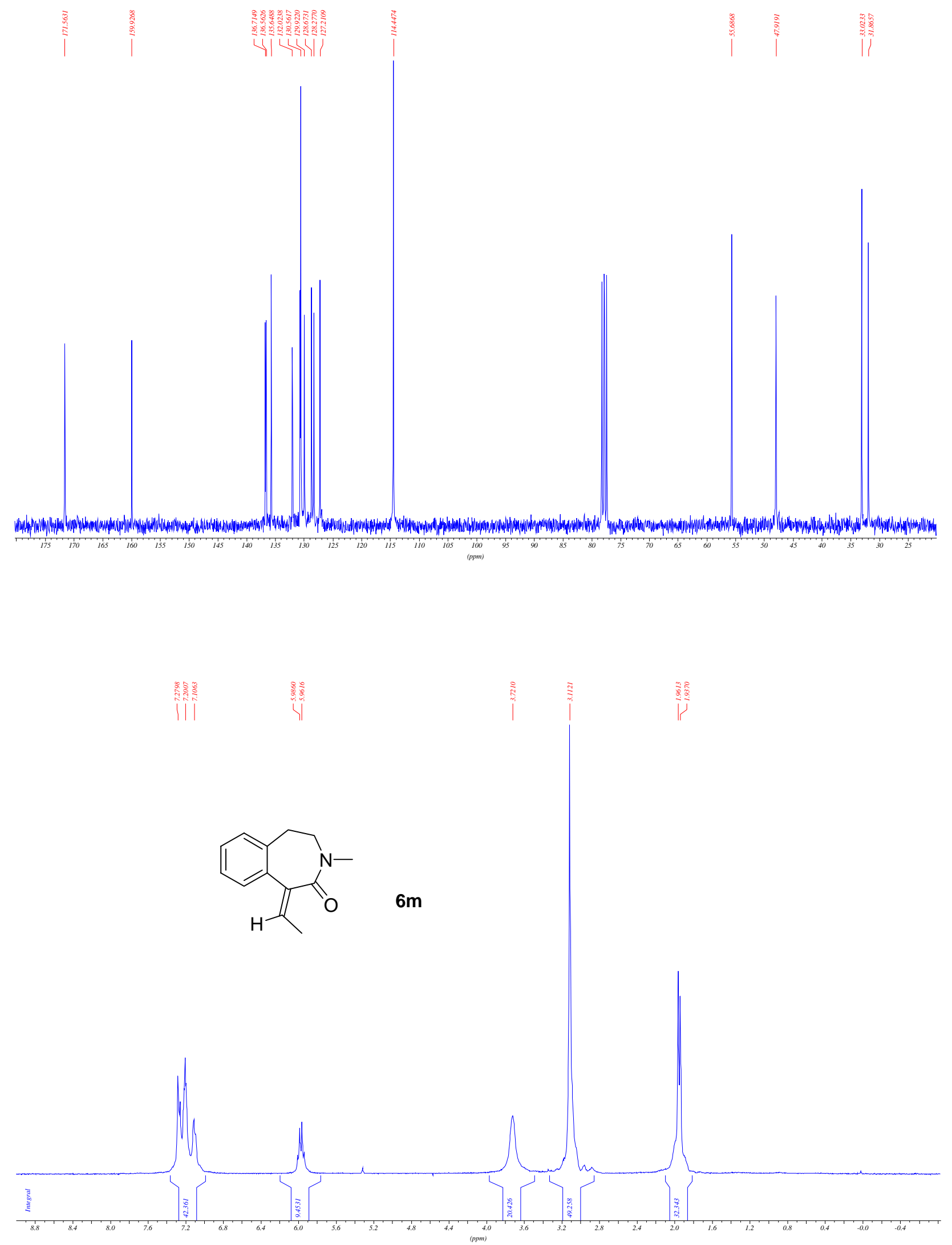



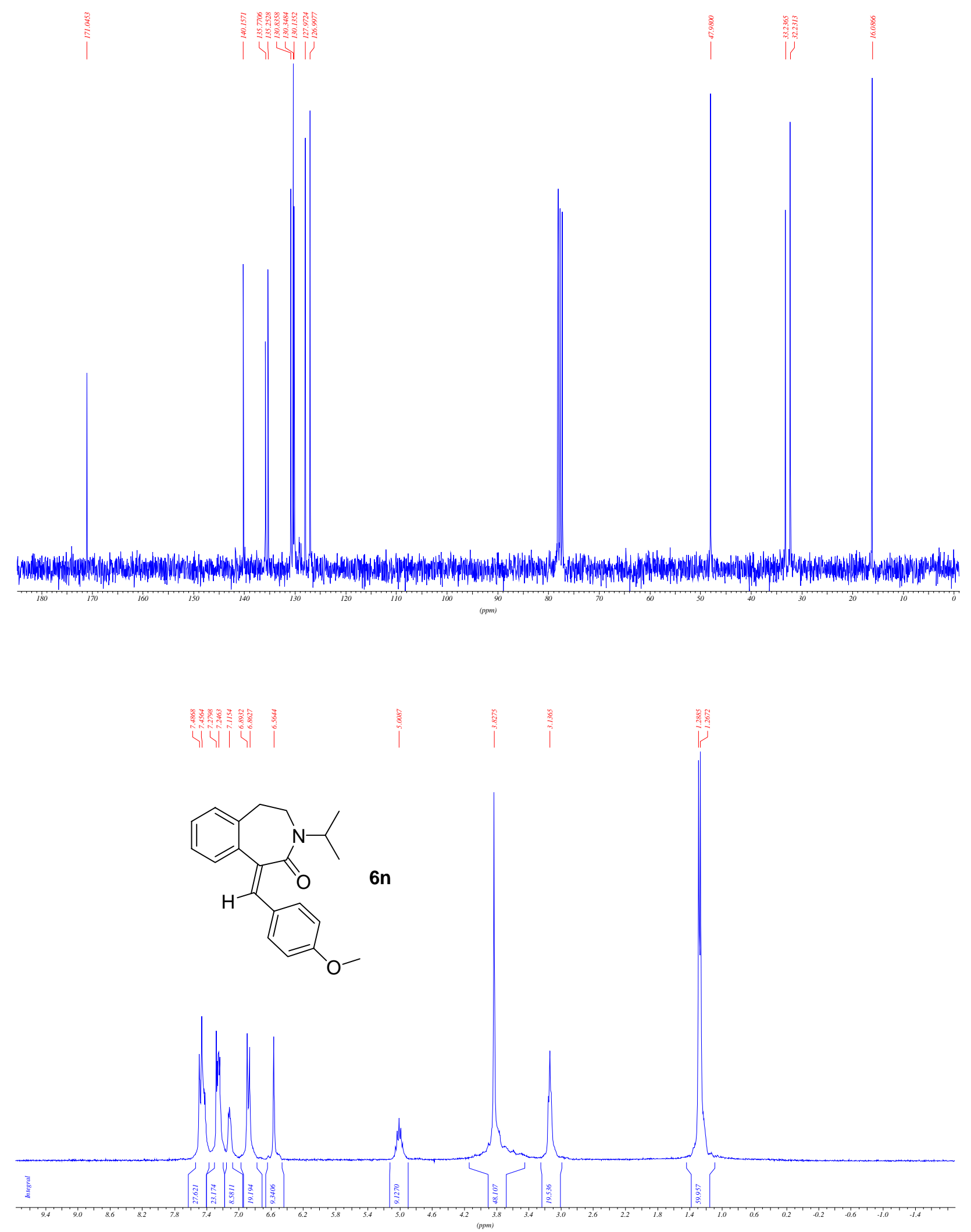

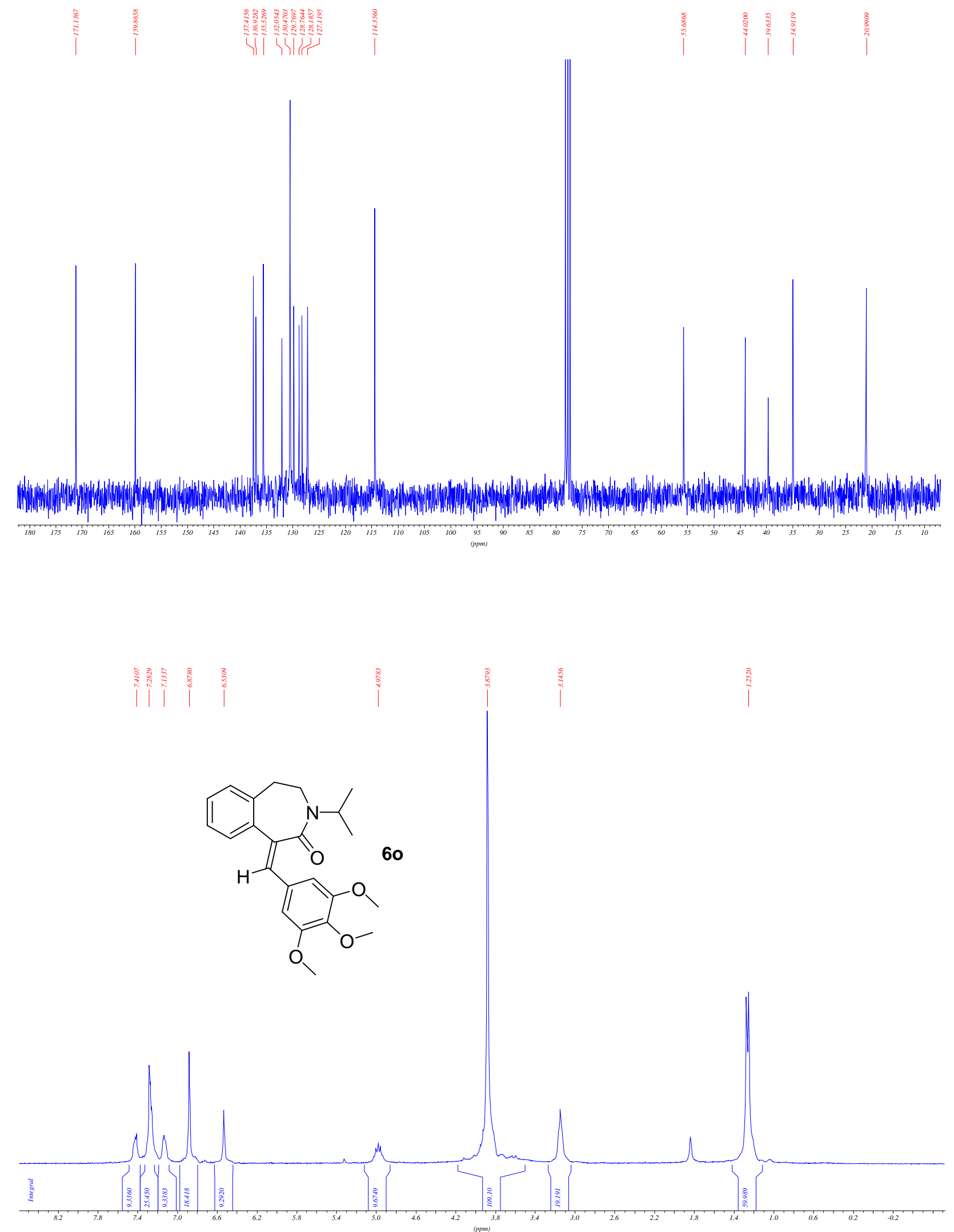

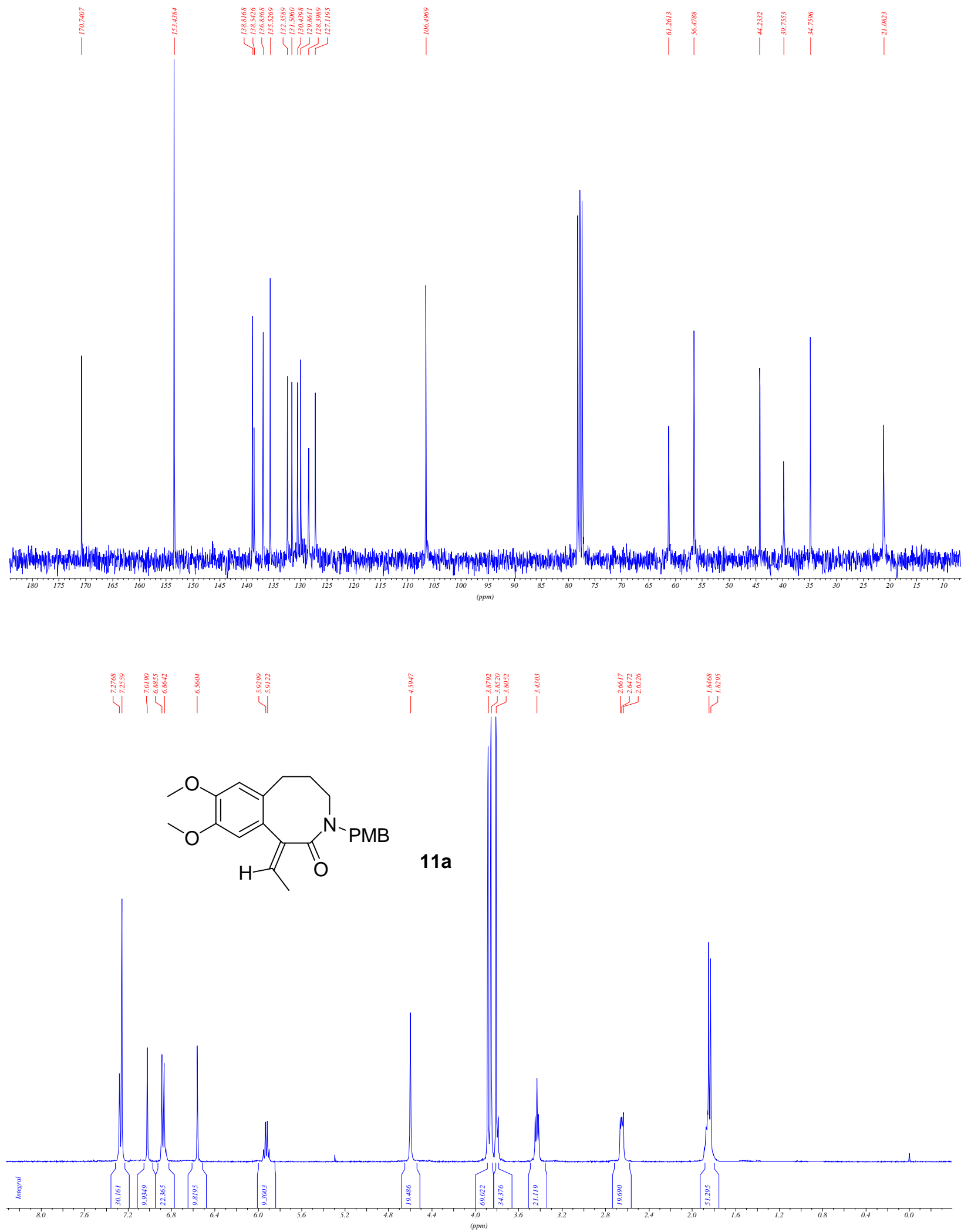

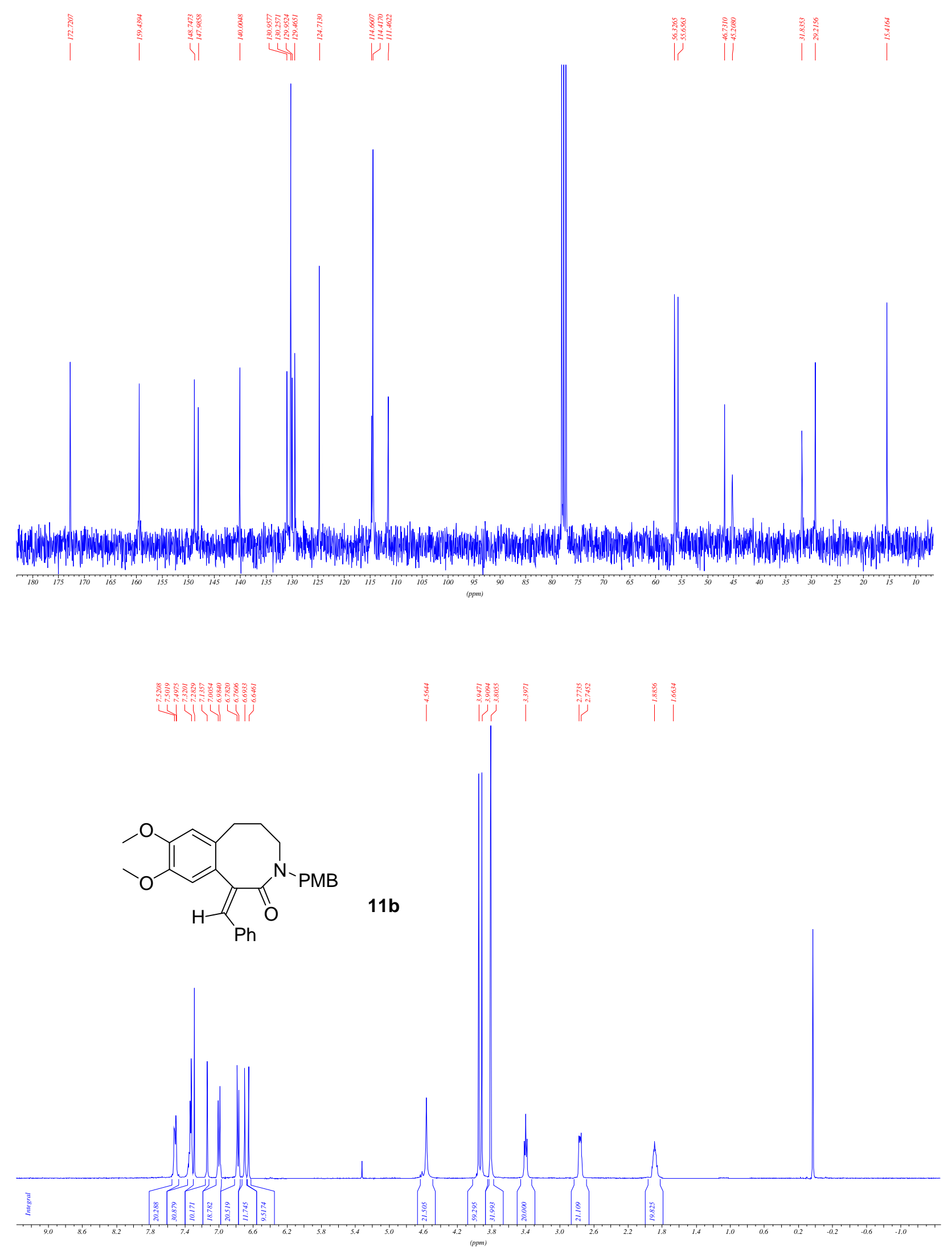

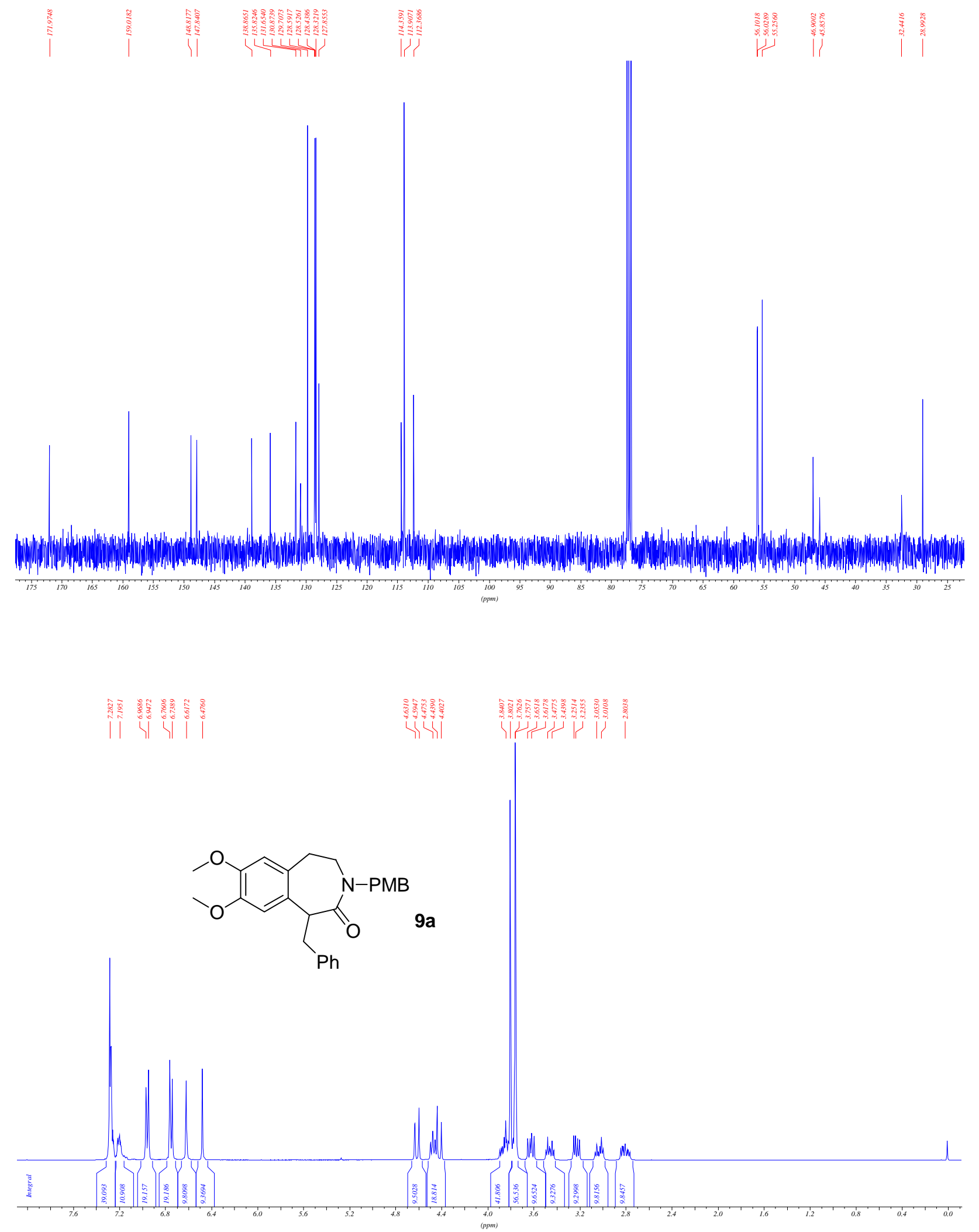

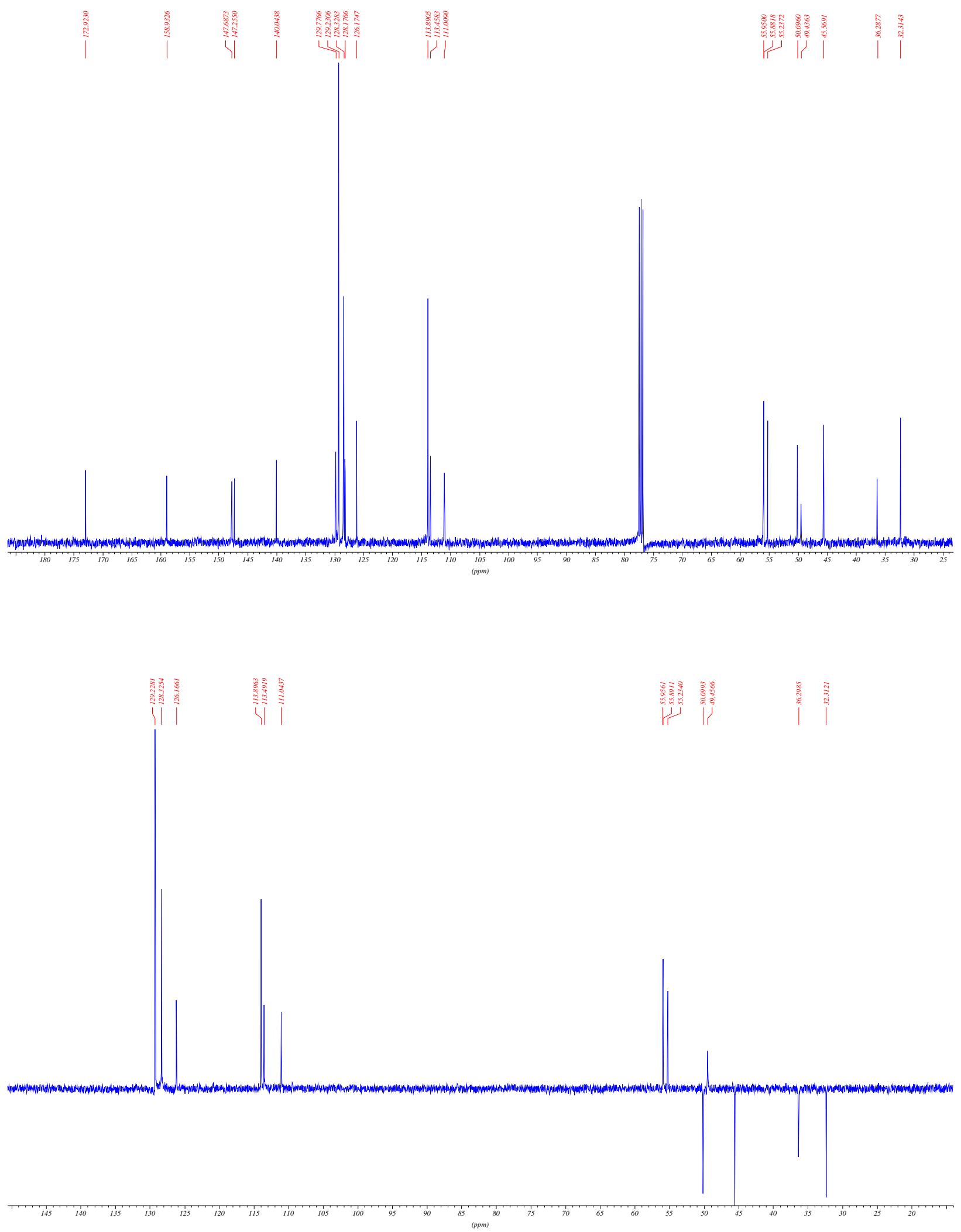

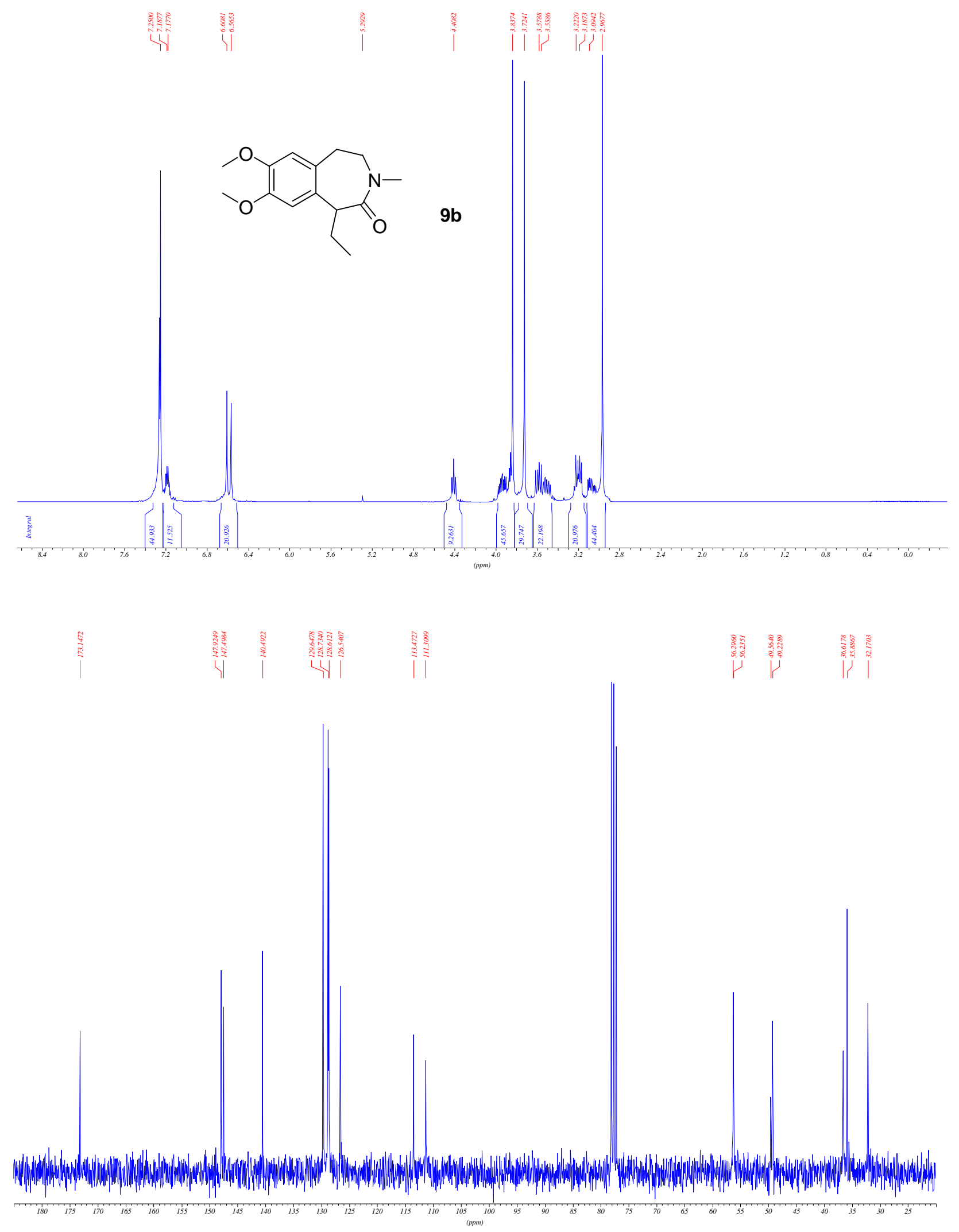


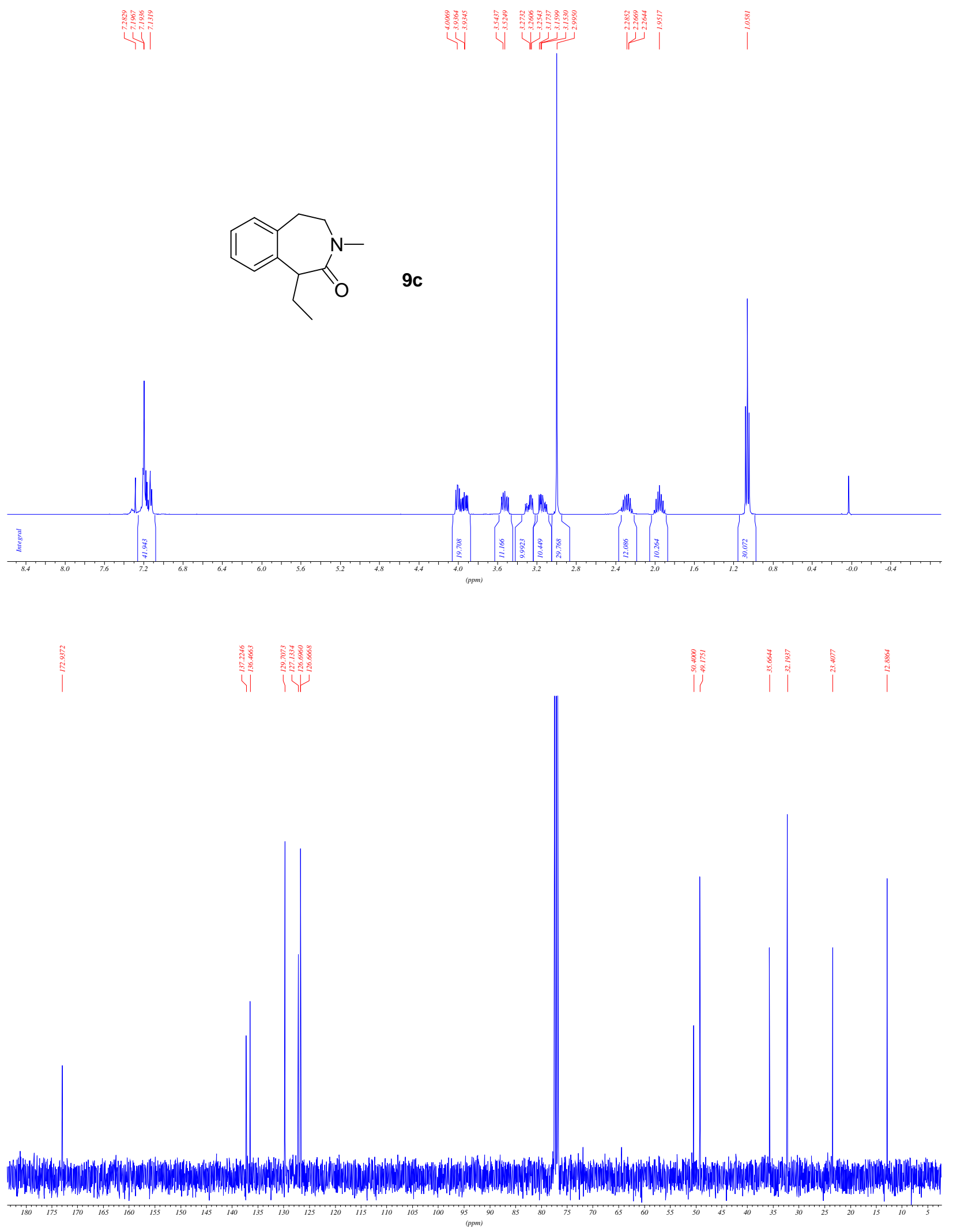



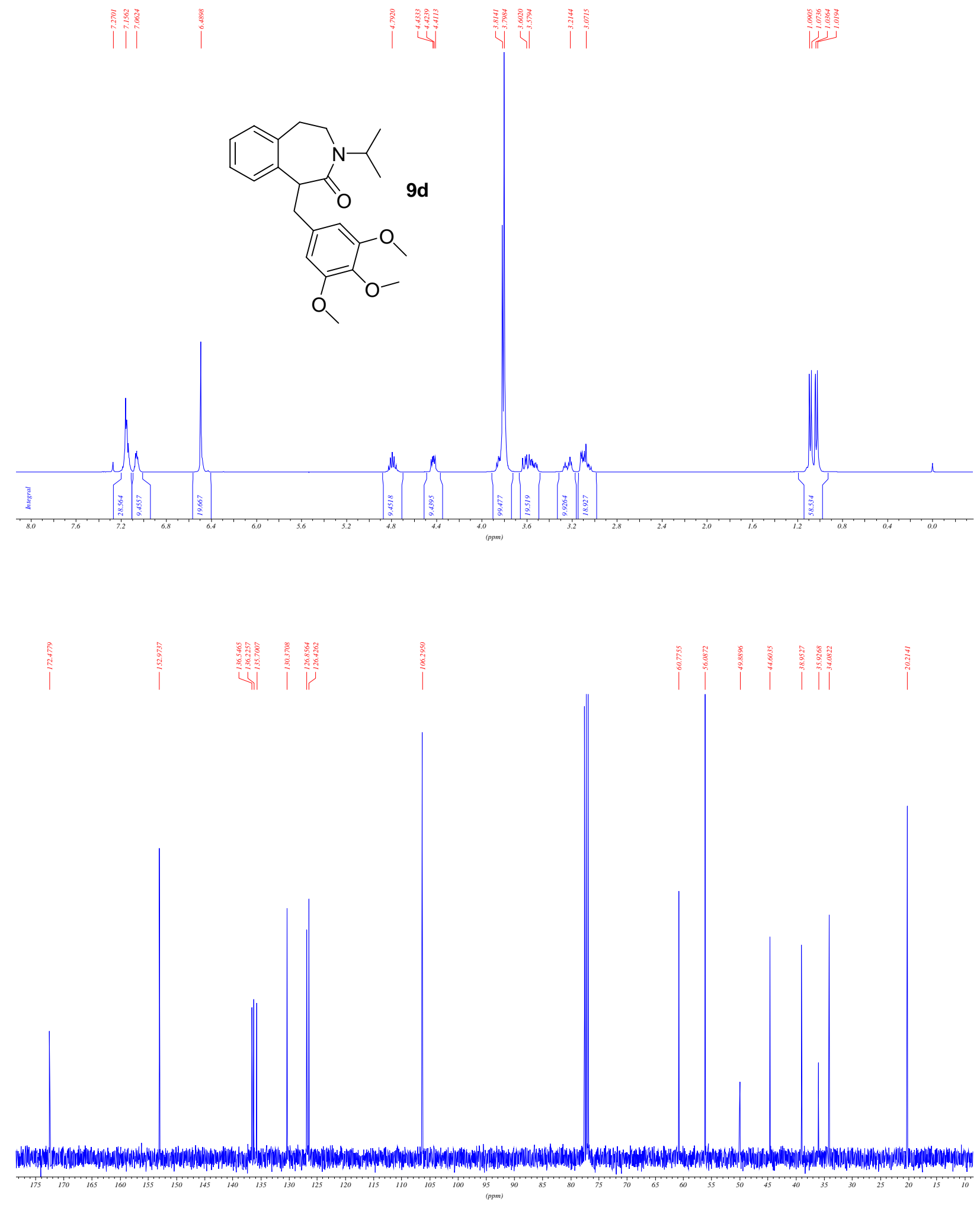